\title{
ENTROPY OF STATIONARY NONEQUILIBRIUM MEASURES OF BOUNDARY DRIVEN SYMMETRIC SIMPLE EXCLUSION PROCESSES
}

\author{
CÉDRIC BERNARDIN AND CLAUDIO LANDIM
}

\begin{abstract}
We examine the entropy of stationary nonequilibrium measures of boundary driven symmetric simple exclusion processes. In contrast with the Gibbs-Shannon entropy 1, 10, the entropy of nonequilibrium stationary states differs from the entropy of local equilibrium states.
\end{abstract}

\section{INTRODUCTION}

In the last decade important progress has been accomplished in the understanding of nonequilibrium stationary states through the study of stochastic lattice gases (4, 8] and references therein).

The simplest nontrivial example of such dynamics is the one-dimensional simple symmetric exclusion process on the finite lattice $\{1,2, \ldots, N-1\}$ with particle reservoirs coupled to the sites 1 and $N-1$. In this model the microscopic states are described by the vector $\eta=(\eta(1), \eta(2), \ldots, \eta(N-1))$, where $\eta(i)=1$ if the site $i$ is occupied and $\eta(i)=0$ if the site is empty. Each particle, independently from the others, perform a nearest-neighbor symmetric random walk with the convention that each time a particle attempts to jump to a site already occupied the jump is suppressed. At the boundaries, particles are created and destroyed in order for the density to be $\alpha$ at the left boundary and $\beta$ at the right boundary, $0 \leq \alpha, \beta \leq 1$.

We denote by $\mu_{\alpha, \beta}^{N}$ the stationary state of this system which is a probability measure in the space of configurations and which can be expressed in terms of a product of matrices [9]. Since the particle number is the only conserved quantity in the bulk, in the scaling limit $N \rightarrow \infty, i / N \rightarrow x \in[0,1]$, the system is described by a single density field $\rho(x), x \in(0,1)$. The typical density profile $\bar{\rho}(x)$ is the stationary solution of a partial differential equation with boundary conditions. In the context of symmetric exclusion processes,

$$
\bar{\rho}(x)=\alpha(1-x)+\beta x .
$$

The nonequilibrium stationary states exhibit long range correlations 13 which are responsible in the large deviations regime for the non locality of the free energy functional 9, 2. More precisely, if $\gamma$ stands for a density profile different from the typical one $\bar{\rho}$, the asymptotic probability of $\gamma$ is exponentially small and given by

$$
\mu_{\alpha, \beta}^{N}[\gamma(\cdot)] \sim e^{-N V_{\alpha, \beta}(\gamma)},
$$

where the so called nonequilibrium free energy $V_{\alpha, \beta}$ is a non local functional.

Key words and phrases. Nonequilibrium stationary states, Large deviations, quasi-potential, boundary driven symmetric exclusion processes. 
Since in equilibrium the probability of such large deviations is determined by the induced change in the entropy, it is natural to investigate the entropy of nonequilibrium stationary states.

Denote by $\mathfrak{S}_{N}\left(\nu^{N}\right)$ the Gibbs-Shannon entropy of a state $\nu^{N}$ :

$$
\mathfrak{S}_{N}\left(\nu^{N}\right)=-\sum_{\eta} \nu^{N}(\eta) \log \nu^{N}(\eta)
$$

where the sum is carried over all lattice configurations $\eta$. Recently, Bahadoran [1] proved that for a large class of stochastic lattice gases the Gibbs-Shannon entropy of nonequilibrium stationary states has the same asymptotic behavior as the GibbsShannon entropy of local equilibrium states. In our context of boundary driven symmetric simple exclusion processes this result can be stated as follows. Denote by $\nu_{\alpha, \beta}^{N}$ the product measure

$$
\nu_{\alpha, \beta}^{N}(\eta)=\prod_{i=1}^{N-1} \bar{\rho}(i / N)^{\eta(i)}[1-\bar{\rho}(i / N)]^{1-\eta(i)} .
$$

Thus, at site $i$, independently from the other sites, we place a particle with probability $\bar{\rho}(i / N)$ and leave the site empty with probability $1-\bar{\rho}(i / N)$. Bahadoran proved that

$$
\lim _{N \rightarrow \infty} \frac{1}{N} \mathfrak{S}_{N}\left(\mu_{\alpha, \beta}^{N}\right)=\lim _{N \rightarrow \infty} \frac{1}{N} \mathfrak{S}_{N}\left(\nu_{\alpha, \beta}^{N}\right)
$$

The long range correlations of the nonequilibrium stationary state is therefore not captured by the Gibbs-Shannon entropy.

Derrida, Lebowitz and Speer [10] showed that for the symmetric simple exclusion process the difference

$$
\mathfrak{S}_{N}\left(\mu_{\alpha, \beta}^{N}\right)-\mathfrak{S}_{N}\left(\nu_{\alpha, \beta}^{N}\right)
$$

converges as $N \rightarrow \infty$, and that the limit depends on the two points correlation functions. Hence, the long range correlations appear in the first order correction to the Gibbs-Shannon entropy.

In this article we examine the entropy of the stationary nonequilibrium states $\mu_{\alpha, \beta}^{N}$. In the classical Boltzmann-Gibbs theory of equilibrium statistical mechanics [12, the steady state $\mu_{\beta}^{N}(\eta)$ of a microstate $\eta$ is given by

$$
\mu_{\beta}^{N}(\eta)=\frac{1}{Z_{N}(\beta)} \exp (-\beta H(\eta))
$$

where $\beta$ is the inverse of the temperature, $H(\eta)$ the energy of $\eta$ and $Z_{N}(\beta)$ the partition function. The Boltzmann entropy is then defined as the limit, when the degrees of freedom $N$ of the system converges to infinity, of $1 / N$ times the logarithm of the number of microstates with a prescribed energy:

$$
S(E)=\lim _{\delta \rightarrow 0} \lim _{N \rightarrow \infty} N^{-1} \log \left(\sum_{\eta} \mathbf{1}\{|H(\eta)-N E| \leq \delta N\}\right)
$$

where the summation is performed over all configurations $\eta$ and where $\mathbf{1}\{A\}$ is the indicator of the set $A$. The pressure $P(\beta)$ is defined by

$$
P(\beta)=\lim _{N \rightarrow \infty} \frac{1}{N \beta} \log Z_{N}(\beta)
$$


and the Boltzmann entropy is related to the pressure function by

$$
S(E)=\inf _{\beta>0}\{\beta P(\beta)+\beta E\} .
$$

In view of (1.1) and by analogy, we define the energy of a microstate $\eta$ as $-\log \mu_{\alpha, \beta}^{N}(\eta)$ and the entropy of the stationary nonequilibrium measure $\mu_{\alpha, \beta}^{N}$ by

$$
S_{\alpha, \beta}(E)=\lim _{\delta \rightarrow 0} \lim _{N \rightarrow \infty} N^{-1} \log \left(\sum_{\eta \in \Omega_{N}} 1\left\{\left|N^{-1} \log \mu_{\alpha, \beta}^{N}(\eta)+E\right| \leq \delta\right\}\right) .
$$

We propose in (2.5) a variational formula for the entropy function $S_{\alpha, \beta}$ in terms of the nonequilibrium free energy $V_{\alpha, \beta}$ and the equilibrium Gibbs-Shannon entropy, that we conjecture to be valid for a large class of boundary driven stochastic lattice gases. This formula is based on a strong form of local equilibrium, stated as assumption $(\mathbf{H})$. We present in (2.9) an explicit formula for the entropy function $S_{\alpha, \beta}$ and we show in 2.13) that it is strictly concave, being the Legendre transform of a strictly concave function $P_{\alpha, \beta}$, identified as the nonequilibrium pressure. This last point is proved in section 4 .

In Section 3 we compute the entropy of stationary nonequilibrium measures of boundary driven zero range processes and in Section 7 we show that the entropy of the nonequilibrium stationary states $\mu_{\alpha, \beta}^{N}$ is different from the entropy of the local equilibrium states $\nu_{\alpha, \beta}^{N}$. In Section 5 , we determine the energy band and describe the density profiles with lowest and largest energy. In Section [6, we examine the isentropic surfaces and in the appendix we show that the strong form of local

equilibrium holds for the symmetric simple exclusion process by using the ideas of 9].

\section{StATIONARY NONEQUILIBRIUM ENTROPY FUNCTION}

Fix an integer $N \geq 1,0<\alpha \leq \beta<1$ and let $\Lambda_{N}:=\{1, \ldots, N-1\}$. Denote by $\Omega_{N}:=\{0,1\}^{\Lambda_{N}}$ the configuration space and by $\eta$ the elements of $\Omega_{N}$, so that $\eta(x)=1$, resp. 0 , if site $x$ is occupied, resp. empty, for the configuration $\eta$. We denote by $\sigma^{x, y} \eta$ the configuration obtained from $\eta$ by exchanging the occupation variables $\eta(x)$ and $\eta(y)$, i.e.

$$
\left(\sigma^{x, y} \eta\right)(z):= \begin{cases}\eta(y) & \text { if } z=x \\ \eta(x) & \text { if } z=y \\ \eta(z) & \text { if } z \neq x, y\end{cases}
$$

and by $\sigma^{x} \eta$ the configuration obtained from $\eta$ by flipping the configuration at $x$, i.e.

$$
\left(\sigma^{x} \eta\right)(z):= \begin{cases}1-\eta(x) & \text { if } z=x \\ \eta(z) & \text { if } z \neq x\end{cases}
$$

The one-dimensional boundary driven symmetric exclusion process is the Markov process on $\Omega_{N}$ whose generator $L_{N}$ can be decomposed as

$$
L_{N}=L_{0, N}+L_{-, N}+L_{+, N}
$$


where the generators $L_{0, N}, L_{-, N}, L_{+, N}$ act on functions $f: \Omega_{N} \rightarrow \mathbb{R}$ as

$$
\begin{aligned}
& \left(L_{0, N} f\right)(\eta)=\sum_{x=1}^{N-2}\left[f\left(\sigma^{x, x+1} \eta\right)-f(\eta)\right] \\
& \left(L_{-, N} f\right)(\eta)=\{\alpha[1-\eta(1)]+(1-\alpha) \eta(1)\}\left[f\left(\sigma^{1} \eta\right)-f(\eta)\right] \\
& \quad\left(L_{+, N} f\right)(\eta)=\{\beta[1-\eta(N-1)]+(1-\beta) \eta(N-1)\}\left[f\left(\sigma^{N-1} \eta\right)-f(\eta)\right] .
\end{aligned}
$$

We denote by $\eta_{t}$ the Markov process on $\Omega_{N}$ with generator $L_{N}$. Since the Markov process $\eta_{t}$ is irreducible, for each $N \geq 1$, and $0<\alpha \leq \beta<1$ there exists a unique stationary state denoted by $\mu_{\alpha, \beta}^{N}$.

The entropy function $S_{\alpha, \beta}: \mathbb{R}_{+} \rightarrow\{-\infty\} \cup[0, \log 2]$ associated to the nonequilibrium stationary state $\mu_{\alpha, \beta}^{N}$ is defined by

$$
S_{\alpha, \beta}(E)=\lim _{\delta \rightarrow 0} \lim _{N \rightarrow \infty} \frac{1}{N} \log \sum_{\eta \in \Omega_{N}} \mathbf{1}\left\{\left|N^{-1} \log \mu_{\alpha, \beta}^{N}(\eta)+E\right| \leq \delta\right\}
$$

whenever the limits exist. To keep notation simple, we sometimes denote $S_{\alpha, \beta}$ by $S$.

Note that we may include in the $\operatorname{sum} \mu_{\alpha, \beta}^{N}(\eta)$ :

$$
S_{\alpha, \beta}(E)=E+\lim _{\delta \rightarrow 0} \lim _{N \rightarrow \infty} \frac{1}{N} \log \mu_{\alpha, \beta}^{N}\left\{\left|N^{-1} \log \mu_{\alpha, \beta}^{N}(\eta)+E\right| \leq \delta\right\} .
$$

In particular,

$$
J_{\alpha, \beta}(E)=E-S_{\alpha, \beta}(E)
$$

is the large deviations rate function of the random variables $-N^{-1} \log \mu_{\alpha, \beta}^{N}(\eta)$ under the probability measure $\mu_{\alpha, \beta}^{N}$.

At equilibrium $\alpha=\beta$, the stationary state $\mu_{\alpha, \beta}^{N}$ is a Bernoulli product measure with density $\alpha$ and the entropy function is given by

$$
S_{\alpha}(E):=S_{\alpha, \alpha}(E)=-s\left(-\frac{E+\log (1-\alpha)}{\log \alpha-\log (1-\alpha)}\right),
$$

where

$$
s(\theta)=\theta \log \theta+(1-\theta) \log (1-\theta)
$$

represents the Gibbs-Shannon entropy. This formula is valid for $E$ in the energy band $\left[E_{-}(\alpha), E_{+}(\alpha)\right]$ where

$$
E_{-}(\alpha)=-\log \{\max (\alpha, 1-\alpha)\}, \quad E_{+}(\alpha)=-\log \{\min (\alpha, 1-\alpha)\} .
$$

In the case $\alpha=1 / 2$ the energy band is reduced to the point $\log 2$ and $S_{1 / 2}(\log 2)=$ $\log 2$. Outside the energy band we have $S_{\alpha}(E)=-\infty$.

Identity (2.3) can be derived from the large deviations principle for the random variable $-N^{-1} \log \mu_{\alpha, \alpha}^{N}(\eta)$, which in the equilibrium case is an average of i.i.d. random variables.

Denote by $\langle\cdot, \cdot\rangle$ the scalar product in $L^{2}([0,1])$. Let $\mathcal{M}$ be the set of measurable profiles $m:[0,1] \rightarrow[0,1]$ equipped with the topology induced by weak convergence, namely $m_{n} \rightarrow m$ in $\mathcal{M}$ if and only $\left\langle m_{n}, G\right\rangle \rightarrow\langle m, G\rangle$ for every continuous function $G:[0,1] \rightarrow \mathbb{R}$. For every $m \in \mathcal{M}$ the nonequilibrium free energy [9, 2] $V_{\alpha, \beta}(m)$ of $m$ is defined by

$$
V_{\alpha, \beta}(m)=\int_{0}^{1}\left\{m(x) \log \frac{m(x)}{F(x)}+(1-m(x)) \log \frac{1-m(x)}{1-F(x)}+\log \frac{F^{\prime}(x)}{\beta-\alpha}\right\} d x,
$$


where $F \in C^{1}([0,1])$ is the unique increasing solution of the non linear boundary value problem

$$
\left\{\begin{array}{l}
F^{\prime \prime}=(m-F) \frac{\left(F^{\prime}\right)^{2}}{F(1-F)}, \\
F(0)=\alpha, \quad F(1)=\beta .
\end{array}\right.
$$

To keep notation simple we frequently denote $V_{\alpha, \beta}$ by $V$.

Decompose the set $\Lambda_{N}$ into $r=\varepsilon^{-1}$ adjacent intervals $K_{1}, \ldots, K_{\varepsilon^{-1}}$ of size $\varepsilon N$ and denote by $\mathbf{M}=\left(M_{1}, \ldots, M_{r}\right)$ the number of particles in each box. Let

$$
\nu\left(M_{1}, \ldots, M_{r}\right)=\sum_{\eta \in \Omega_{N}} \mathbf{1}\left\{\sum_{x \in K_{1}} \eta(x)=M_{1}, \ldots, \sum_{x \in K_{r}} \eta(x)=M_{r}\right\} \mu_{\alpha, \beta}^{N}(\eta)
$$

be the probability to find $M_{j}$ particles in the interval $K_{j}, 1 \leq j \leq r$. Denote by $\mu_{\alpha, \beta}^{N}(\cdot \mid \mathbf{M})$ the probability measure $\mu_{\alpha, \beta}^{N}$ conditioned to have $M_{j}$ particles in $K_{j}$, $j=1, \ldots, r$. The set of configurations $\eta$ such that $\sum_{x \in K_{j}} \eta(x)=M_{j}, 1 \leq j \leq r$, is denoted by $\Omega_{N}(\mathbf{M})$ and its cardinality by $Z_{N}(\mathbf{M})$. We shall assume that for every $0<\alpha \leq \beta<1$,

$$
\lim _{\varepsilon \rightarrow 0} \limsup _{N \rightarrow \infty} \sup _{\mathbf{M}} \sup _{\eta \in \Omega_{N}(\mathbf{M})} \frac{1}{N}\left|\log \left\{Z_{N}(\mathbf{M}) \mu_{\alpha, \beta}^{N}(\eta \mid \mathbf{M})\right\}\right|=0 .
$$

We present in the appendix a formal derivation of this hypothesis.

Assumption $(\mathbf{H})$ states that the stationary state $\mu_{\alpha, \beta}^{N}$ conditioned on the number of particles on macroscopic intervals is uniformly close in a logarithmic sense to the uniform measure as the number of intervals increases. As we shall see, this alternative formulation of local equilibrium plays a central role in the investigation of the entropy of stationary nonequilibrium measures. The first main result of this article provides a variational formula for the entropy function. We claim that for every $0<\alpha \leq \beta<1, E \geq 0$,

$$
S_{\alpha, \beta}(E)=\sup _{m \in \mathcal{M}}\left\{\mathbb{S}(m): V_{\alpha, \beta}(m)+\mathbb{S}(m)=E\right\},
$$

where

$$
\mathbb{S}(m)=-\int_{0}^{1} s(m(x)) d x
$$

This formula is a straightforward consequence of assumption $(\mathbf{H})$ and the large deviations for the nonequilibrium stationary state $\mu_{\alpha, \beta}^{N}$. Indeed, we may rewrite $\mu_{\alpha, \beta}^{N}(\eta)$ as $\mu_{\alpha, \beta}^{N}(\eta \mid \mathbf{M}) \nu(\mathbf{M})$. Hence, by definition of the entropy and by assumption $(\mathbf{H})$,

$S(E)=\lim _{\delta \rightarrow 0} \lim _{\varepsilon \rightarrow 0} \lim _{N \rightarrow \infty} \frac{1}{N} \log \sum_{\eta \in \Omega_{N}} \mathbf{1}\left\{\left|N^{-1} \log \nu(\mathbf{M})-N^{-1} \log Z_{N}(\mathbf{M})+E\right| \leq \delta\right\}$.

The previous sum can be rewritten as

$$
\sum_{\mathbf{M}} Z_{N}(\mathbf{M}) \mathbf{1}\left\{\left|N^{-1} \log \nu(\mathbf{M})-N^{-1} \log Z_{N}(\mathbf{M})+E\right| \leq \delta\right\} .
$$


Recall that $N^{-1} \log \nu(\mathbf{M}) \sim-V(m)$ and that $N^{-1} \log Z_{N}(\mathbf{M}) \sim \mathbb{S}(m)$ where $m(\cdot)$ is the macroscopic profile associated to $\mathbf{M}$ :

$$
m=\sum_{i=1}^{r} \rho_{i} \mathbf{1}\left\{\left[x_{i}, x_{i+1}\right)\right\}, \quad \rho_{i}=M_{i} /(N \varepsilon), \quad K_{i}=\left\{\left[N x_{i}\right], \ldots,\left[N x_{i+1}\right]-1\right\} .
$$

Since for a fixed $\varepsilon$ the sum over $\mathbf{M}$ has only a polynomial number of terms in $N$ and since $Z_{N}(\mathbf{M})$ is exponentially large in $N$, only the term which maximizes $Z_{N}(\mathbf{M})$ contributes. The result follows.

Note that by [3, Theorem 4.1], the functional $V_{\alpha, \beta}+\mathbb{S}$ is continuous in $\mathcal{M}$.

2.1. The nonequilibrium pressure. Let $A: \mathbb{R}_{*} \rightarrow \mathbb{R}_{+}, P: \mathbb{R} \rightarrow \mathbb{R}$ be given by

$$
\begin{gathered}
A(\theta)=A_{\alpha, \beta}(\theta):=\int_{\alpha}^{\beta} \frac{d x}{\left[x^{\theta}+(1-x)^{\theta}\right]^{1 / \theta}}, \quad \theta \neq 0, \\
P(\theta)=P_{\alpha, \beta}(\theta):=\theta \log \left(\frac{A_{\alpha, \beta}(\theta)}{\beta-\alpha}\right), \quad \theta \neq 0,
\end{gathered}
$$

$P(0)=-\log 2$. As we shall see in (2.13), $P$ is the Legendre transform of the entropy function $S$ and may thus be identified with the nonequilibrium pressure. An elementary computation shows that $\log A$ is strictly increasing on the intervals $(-\infty, 0)$ and $(0, \infty)$ and that $\lim _{\theta \rightarrow \pm 0} \log A(\theta)=\mp \infty$. Moreover,

$$
P^{\prime}(\theta)=\frac{1}{A(\theta) \theta^{2}} \int_{(\alpha /(1-\alpha))^{\theta}}^{(\beta /(1-\beta))^{\theta}} \frac{1}{1+x} \frac{(1+x) \log (1+x)-x \log x}{\left(x^{1 / \theta}+1\right)\left(x^{\theta-1}+x^{\theta}\right)^{1 / \theta}} d x+\log \frac{A(\theta)}{\beta-\alpha}
$$

for $\theta \neq 0$, and

$$
P^{\prime}(0)=\log \left(\frac{1}{\beta-\alpha} \int_{\alpha}^{\beta} \frac{d x}{\sqrt{x(1-x)}}\right) .
$$

We prove in Section 4 the following properties.

Lemma 2.1. The function $P$ is a $C^{2}$ strictly concave function. Moreover,

$$
\lim _{\theta \rightarrow \pm \infty}\left\{P(\theta)-\theta E_{\mp}\right\}=0, \quad \lim _{\theta \rightarrow \pm \infty}\left\{P(\theta)-\theta P^{\prime}(\theta)\right\}=0,
$$

where

$$
\begin{gathered}
E_{+}=E_{+}(\alpha, \beta)=\log \left(\frac{1}{\beta-\alpha} \int_{\alpha}^{\beta} \frac{d x}{\min \{x, 1-x\}}\right) \\
E_{-}=E_{-}(\alpha, \beta)=\log \left(\frac{1}{\beta-\alpha} \int_{\alpha}^{\beta} \frac{d x}{\max \{x, 1-x\}}\right) .
\end{gathered}
$$

It follows from this lemma that $\lim _{\theta \rightarrow \pm \infty} \theta\left\{P^{\prime}(\theta)-E_{\mp}\right\}=0$, and that for each $E \in\left(E_{-}, E_{+}\right)$, there exists a unique $\theta_{E}=\theta(\alpha, \beta, E) \in \mathbb{R}$ such that

$$
P^{\prime}\left(\theta_{E}\right)=E \text {. }
$$

Define the functions $\gamma_{ \pm}: \mathbb{R} \rightarrow \mathbb{R}_{+}$by

$$
\begin{gathered}
\gamma_{-}(\theta)=\gamma_{-}(\alpha, \beta, \theta)=\min \left\{\left(\frac{\alpha}{1-\alpha}\right)^{\theta},\left(\frac{\beta}{1-\beta}\right)^{\theta}\right\}, \\
\gamma_{+}(\theta)=\gamma_{+}(\alpha, \beta, \theta)=\max \left\{\left(\frac{\alpha}{1-\alpha}\right)^{\theta},\left(\frac{\beta}{1-\beta}\right)^{\theta}\right\},
\end{gathered}
$$


and let $W_{E}=W_{\alpha, \beta, E}:\left[\gamma_{-}\left(\theta_{E}\right), \gamma_{+}\left(\theta_{E}\right)\right] \rightarrow[0,1]$ be the monotone function given by

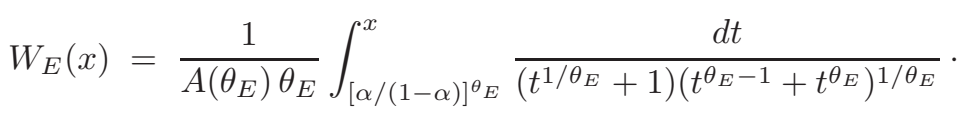

Clearly, $W_{E}\left([\alpha /(1-\alpha)]^{\theta_{E}}\right)=0$. On the other hand, the change of variables $t=$ $[x /(1-x)]^{\theta_{E}}$ shows that $W_{E}\left([\beta /(1-\beta)]^{\theta_{E}}\right)=1$ in view of the definition of $A(\theta)$. Let $h_{E}:[0,1] \rightarrow\left[\gamma_{-}\left(\theta_{E}\right), \gamma_{+}\left(\theta_{E}\right)\right]$ be the inverse of $W_{E}$ so that $h_{E}(0)=(\alpha /(1-\alpha))^{\theta_{E}}$, $h_{E}(1)=(\beta /(1-\beta))^{\theta_{E}}$.

2.2. An explicit formula for $S_{\alpha, \beta}$. We are now in a position to present an explicit formula for the entropy function $S$. We claim that for every $0<\alpha \leq \beta<1$, $E_{-}(\alpha, \beta)<E<E_{+}(\alpha, \beta)$,

$$
S_{\alpha, \beta}(E)=\mathbb{S}\left(\frac{h_{E}(x)}{1+h_{E}(x)}\right) .
$$

Indeed, consider the variational problem (2.5). Let $\theta$ be the Lagrange multiplier and let $R(m, \theta)$ be the function defined by

$$
R(m, \theta)=\mathbb{S}(m)-\theta\{V(m)+\mathbb{S}(m)-E\} .
$$

Since by [9, 2] $(\delta V / \delta m)=\log [m /(1-m)]-\log [F /(1-F)]$, the conditions $(\delta R / \delta m)=$ $\partial_{\theta} R=0$ imply that

$$
\begin{gathered}
m=\frac{(F /[1-F])^{\theta}}{1+(F /[1-F])^{\theta}} \\
\int_{0}^{1}\left\{m(x) \log F(x)+[1-m(x)] \log [1-F(x)]-\log \left(\frac{F^{\prime}(x)}{\beta-\alpha}\right)\right\} d x=-E,
\end{gathered}
$$

where $F$ is the unique increasing solution of the non linear boundary value problem (2.4). We report the first identity in (2.10) to (2.4) to get that

$$
\frac{F^{\prime \prime}}{F^{\prime}}=\left\{\frac{F^{\theta-1}-(1-F)^{\theta-1}}{(1-F)^{\theta}+F^{\theta}}\right\} F^{\prime} .
$$

Since $d / d z\left[\theta^{-1} \log \left((1-z)^{\theta}+z^{\theta}\right)\right]=\frac{z^{\theta-1}-(1-z)^{\theta-1}}{(1-z)^{\theta}+z^{\theta}}$ and $\left(\log F^{\prime}\right)^{\prime}=F^{\prime \prime} / F^{\prime}$ we deduce from the previous equation that

$$
F^{\prime}=A\left[(1-F)^{\theta}+F^{\theta}\right]^{1 / \theta}
$$

for some positive constant $A$ determined by the boundary conditions satisfied by F:

$$
A=\int_{0}^{1} \frac{F^{\prime}(x)}{\left[(1-F)^{\theta}+F^{\theta}\right]^{1 / \theta}} d x
$$

The change of variables $y=F(x)$ shows that $A=A(\theta)$ is given by (2.6).

Recall the definition of $\gamma_{ \pm}(\theta)$. Let $g_{\theta}:[0,1] \rightarrow\left[\gamma_{-}(\theta), \gamma_{+}(\theta)\right]$ be given by $g_{\theta}=(F /(1-F))^{\theta}$ and observe that

$$
g_{\theta}^{\prime}=A(\theta) \theta\left(g_{\theta}^{1 / \theta}+1\right)\left(g_{\theta}^{\theta-1}+g_{\theta}^{\theta}\right)^{1 / \theta} .
$$


Define $U_{\theta}:\left[\gamma_{-}(\theta), \gamma_{+}(\theta)\right] \rightarrow[0,1]$ by

$$
U_{\theta}(x)=\frac{1}{A(\theta) \theta} \int_{(\alpha /(1-\alpha))^{\theta}}^{x} \frac{d t}{\left(t^{1 / \theta}+1\right)\left(t^{\theta-1}+t^{\theta}\right)^{1 / \theta}}
$$

and remark that $g_{\theta}=U_{\theta}^{-1}$.

In the second equation of (2.10) replacing $m$ by $g_{\theta} /\left(1+g_{\theta}\right)$ and $F^{\prime}$ by the right hand side of identity (2.11), we obtain that

$$
\frac{1}{\theta} \int_{0}^{1}\left\{\frac{g_{\theta}(x)}{1+g_{\theta}(x)} \log g_{\theta}(x)-\log \left(1+g_{\theta}(x)\right)\right\} d x-\log \frac{A(\theta)}{\beta-\alpha}=-E .
$$

Performing the change of variables $y=g_{\theta}(x)$, we get that

$$
\frac{1}{A(\theta) \theta^{2}} \int_{(\alpha /(1-\alpha))^{\theta}}^{(\beta /(1-\beta))^{\theta}} \frac{1}{1+x} \frac{x \log x-(1+x) \log (1+x)}{\left(x^{1 / \theta}+1\right)\left(x^{\theta-1}+x^{\theta}\right)^{1 / \theta}} d x-\log \frac{A(\theta)}{\beta-\alpha}=-E .
$$

In view of the explicit expression for $P^{\prime}$, we may rewrite the previous identity as $P^{\prime}(\theta)=E$. Therefore, by (2.8), $\theta=\theta_{E}$, and hence $U_{\theta}=W_{E}, g_{\theta}=h_{E}$. Moreover, in view of (2.10), the density profile $m$ which solves the variational problem (2.5) is $m=h_{E} /\left[1+h_{E}\right]$. This proves (2.9).

2.3. A variational formula for $S_{\alpha, \beta}$. We conclude this section showing that $P_{\alpha, \beta}$ is the Legendre transform of $S_{\alpha, \beta}$ and can therefore be identified with the nonequilibrium pressure.

For every $0<\alpha \leq \beta<1, E \geq 0$,

$$
S(E)=\inf _{\theta \in \mathbb{R}}\{\theta E-P(\theta)\} .
$$

If $E$ belongs to the energy band $\left(E_{-}, E_{+}\right)$the infimum is attained at $\theta_{E}$ given by (2.8) and

$$
S(E)=\theta_{E} E-P\left(\theta_{E}\right) \text {. }
$$

Moreover, $S\left(E_{ \pm}\right)=0$ and $S(E)=-\infty$ if $E \notin\left[E_{-}, E_{+}\right]$.

By abuse of notation we shall call $S$ the Legendre transform of $P$. Usually the Legendre transform is defined as a supremum and involves convex functions. However, by taking a minus sign we may transform convex functions into concave functions and supremums into infimums.

The proof of (2.13) is simple. In section 5 we show that $S(E)=-\infty$ outside $\left[E_{-}, E_{+}\right]$and that $S\left(E_{ \pm}\right)=0$. On the other hand, by Lemma 2.1, $\theta E-P(\theta)$ is a monotone non-decreasing function for $E \geq E_{+}$. Hence, for $E \geq E_{+}, \inf _{\theta \in \mathbb{R}}\{\theta E-$ $P(\theta)\}=\lim _{\theta \rightarrow-\infty}\{\theta E-P(\theta)\}$. By Lemma 2.1 again, $\{\theta E-P(\theta)\}$ converges to $-\infty, 0$ for $E>E_{+}, E=E_{+}$, respectively. Therefore, by the first observation of the proof, $S(E)=\inf \{\theta E-P(\theta)\}$ for $E \geq E_{+}$. The case $E \leq E_{-}$is analogous.

By Lemma 2.1 $\lim _{\theta \rightarrow \pm \infty}\{\theta E-P(\theta)\}=+\infty$ for $E \in\left(E_{-}, E_{+}\right)$and the function $\theta \rightarrow \theta E-P(\theta)$ is strictly convex on $\mathbb{R}$. Hence, $\inf \{\theta E-P(\theta)\}=\theta_{E} E-P\left(\theta_{E}\right)$, where $\theta_{E}$ solves (2.8). We may rewrite this expression as $\theta_{E} P^{\prime}\left(\theta_{E}\right)-P\left(\theta_{E}\right)$. In view of (2.7), to conclude the proof of (2.13) it remains to show that the first term on the right hand side of (2.7) multiplied by $\theta$ and computed at $\theta=\theta_{E}$ coincides with (2.9). This can be shown by performing the change of variables $u=h_{E}(x)$ in (2.9) and recalling that $W_{E}$ is the inverse of $h_{E}$.

It follows from (2.13) that $S$ is concave and that $P$, the Legendre transform of the entropy, can be identified with the pressure. 
The equilibrium case can be recovered by letting $\alpha \rightarrow \beta$. In this case,

$$
\begin{aligned}
\theta(\alpha, \alpha, E) & =-\frac{1}{\log [\alpha /(1-\alpha)]} \frac{E+\log (1-\alpha)}{E+\log (\alpha)}, \\
h_{E}(x) & =-\frac{E+\log (1-\alpha)}{E+\log (\alpha)}
\end{aligned}
$$

\section{BOUNDARY DRIVEN ZERO RANGE PROCESSES}

We compute in this section the entropy of stationary nonequilibrium measures of boundary driven zero range processes. The model is described by a positive integer variable $\eta(x)$ representing the number of particles at site $x \in \Lambda_{N}$. The state space $\mathbb{N}^{\Lambda_{N}}$ is denoted $\Omega_{N}$. At exponential times one particle jumps with rate $g(\eta(x))$ to one of the nearest-neighbor sites. The function $g: \mathbb{N} \rightarrow \mathbb{R}_{+}$is increasing and $g(0)=0$. We assume that the system interacts with particle reservoirs at the boundary of $\Lambda_{N}$ whose activity at the right is $\varphi_{+}>0$ and at the left is $\varphi_{-}>0$. The microscopic dynamics is defined by the generator

$$
\mathcal{L}_{N}=\mathcal{L}_{0, N}+\mathcal{L}_{-, N}+\mathcal{L}_{+, N}
$$

where

$$
\begin{gathered}
\mathcal{L}_{0, N} f(\eta)=\sum_{x=1}^{N-2}\left\{g(\eta(x))\left[f\left(T^{x, x+1} \eta\right)-f(\eta)\right]+g(\eta(x+1))\left[f\left(T^{x+1, x} \eta\right)-f(\eta)\right]\right\} \\
\mathcal{L}_{-, N} f(\eta)=g(\eta(1))\left[f\left(S_{1}^{-} \eta\right)-f(\eta)\right]+\varphi_{-}\left[f\left(S_{1}^{+} \eta\right)-f(\eta)\right], \\
\mathcal{L}_{+, N} f(\eta)=g(\eta(N-1))\left[f\left(S_{N-1}^{-} \eta\right)-f(\eta)\right]+\varphi_{+}\left[f\left(S_{N-1}^{+} \eta\right)-f(\eta)\right],
\end{gathered}
$$

in which

$$
\left(T^{x, y} \eta\right)(z)=\left\{\begin{array}{cll}
\eta(z) & \text { if } & z \neq x, y \\
\eta(z)-1 & \text { if } & z=x \\
\eta(z)+1 & \text { if } & z=y
\end{array}\right.
$$

is the configuration obtained from $\eta$ when a particle jumps from $x$ to $y$, and

$$
\left(S_{x}^{ \pm} \eta\right)(z)=\left\{\begin{array}{ccc}
\eta(z) & \text { if } & z \neq x \\
\eta(z) \pm 1 & \text { if } & z=x
\end{array}\right.
$$

is the configuration where we added (resp. subtracted) one particle at $x$. Note that, since $g(0)=0$, the number of particles cannot become negative.

The invariant measures of the boundary driven zero range processes can be computed explicitly. Let $\varphi_{N}: \Lambda_{N} \rightarrow \mathbb{R}_{+}$be the linear interpolation between $\varphi_{-}$ and $\varphi_{+}$:

$$
\varphi_{N}(x)=\left(1-\frac{x}{N}\right) \varphi_{-}+\frac{x}{N} \varphi_{+} .
$$

The invariant measure $m_{\varphi_{-}, \varphi_{+}}^{N}$ is the product measure whose marginals are given by

$$
m_{\varphi_{-}, \varphi_{+}}^{N}\{\eta: \eta(x)=k\}=\frac{1}{Z\left(\varphi_{N}(x)\right)} \frac{\varphi_{N}(x)^{k}}{g(1) \cdots g(k)}, \quad k \geq 0,
$$

where $Z(\varphi)=1+\sum_{k \geq 1} \varphi^{k} /[g(1) \cdots g(k)]$ is the normalization constant. 
Denote by $R: \mathbb{R}_{+} \rightarrow \mathbb{R}_{+}$the density of particle under the stationary state with activity equal to $\varphi$ on both boundaries:

$$
R(\varphi)=E_{m_{\varphi, \varphi}^{N}}[\eta(x)]=\frac{1}{Z(\varphi)} \sum_{k \geq 1} k \frac{\varphi^{k}}{g(1) \cdots g(k)}=\frac{\varphi Z^{\prime}(\varphi)}{Z(\varphi)}
$$

and by $\Phi: \mathbb{R}_{+} \rightarrow \mathbb{R}_{+}$the inverse of $R$ :

$$
\Phi=R^{-1} .
$$

Under the stationary state, the typical density profile $\bar{\rho}:[0,1] \rightarrow \mathbb{R}_{+}$is the unique solution of the elliptic equation

$$
\left\{\begin{array}{l}
\Delta \Phi(\bar{\rho})=0 \\
\bar{\rho}(0)=R\left(\varphi_{-}\right), \bar{\rho}(1)=R\left(\varphi_{+}\right)
\end{array}\right.
$$

where $\Delta$ stands for the Laplacian. As $N \uparrow \infty$, the activity profile $\varphi_{N}$ introduced in (3.1) converges to $\Phi(\bar{\rho})$ :

$$
\lim _{N \rightarrow \infty} \hat{\varphi}_{N}=\Phi(\bar{\rho}),
$$

where $\hat{\varphi}_{N}:[0,1] \rightarrow \mathbb{R}_{+}$is the function defined by $\hat{\varphi}_{N}(0)=\varphi_{-}, \hat{\varphi}_{N}(x / N)=\varphi_{N}(x)$, $x \in \Lambda_{N}, \hat{\varphi}_{N}(1)=\varphi_{+}$and extended to the interval $[0,1]$ by linear interpolation.

The weight of a configuration $\eta$ under the stationary state $m_{\varphi_{-}, \varphi_{+}}^{N}$ is given by

$$
m_{\varphi_{-}, \varphi_{+}}^{N}(\eta)=\exp \sum_{x=1}^{N-1}\left\{\eta(x) \log \varphi_{N}(x)-\log [g(1) \cdots g(\eta(x))]-\log Z\left(\varphi_{N}(x)\right)\right\} .
$$

In the special case where $g(k)=\mathbf{1}\{k \geq 1\}$, the weight $m_{\varphi_{-}, \varphi_{+}}^{N}(\eta)$ is a function of the empirical density. Hence, if in this case we define for a profile $\rho:[0,1] \rightarrow \mathbb{R}_{+}$,

$$
\mathcal{H}_{\varphi_{-}, \varphi_{+}}(\rho):=\int_{0}^{1} \rho(x) \log \Phi(\bar{\rho}(x))-\log Z(\Phi(\bar{\rho}(x))) d x,
$$

we have that

$$
m_{\varphi_{-}, \varphi_{+}}^{N}(\rho) \sim e^{N \mathcal{H}_{\varphi_{-}, \varphi_{+}}(\rho)} .
$$

In general, the weight of a configuration is not a function of the empirical density but a function of the field associated to the variables $\xi(x)=\eta(x)-$ $\log [g(1) \cdots g(\eta(x))] / \log \varphi_{N}(x)$.

The nonequilibrium free energy functional is easy to compute in the context of zero range boundary driven systems since the stationary state is a product measure. A simple computation shows that

$$
V_{\varphi_{-}, \varphi_{+}}(\rho)=\int_{0}^{1} \rho(x) \log \frac{\Phi(\rho(x))}{\Phi(\bar{\rho}(x))}-\log \frac{Z(\Phi(\rho(x)))}{Z(\Phi(\bar{\rho}(x)))} d x .
$$

To present an explicit formula for the entropy function in this context, we need to introduce some notation borrowed from the theory of large deviations of i.i.d. random variables. Fix $\varphi>0$, let $M: \mathbb{R} \rightarrow \mathbb{R}_{+}$be given by

$$
M_{\varphi}(a)=\frac{1}{Z(\varphi)} \sum_{k \geq 0} \frac{\varphi^{k}}{g(1) \cdots g(k)} e^{a F_{\varphi}(k)},
$$

where $F_{\varphi}(k)=k-[\log \varphi]^{-1} \log [g(1) \cdots g(k)]$, and let $R_{\varphi}(a)=M_{\varphi}^{\prime}(a) / M_{\varphi}(a)$. The large deviations rate function $I_{\varphi}: \mathbb{R} \rightarrow \mathbb{R}_{+}$for the mean of the i.i.d. random 
variables $\xi(x)=\eta(x)-[\log \varphi]^{-1} \log [g(1) \cdots g(\eta(x))], 1 \leq x \leq N-1$, distributed according to $m_{\varphi, \varphi}^{N}$ is given by

$$
I_{\varphi}(x)=x R_{\varphi}^{-1}(x)-\log M_{\varphi}\left(R_{\varphi}^{-1}(x)\right) .
$$

In the particular case where $g(k)=\mathbf{1}\{k \geq 1\}$, we get that $Z(\varphi)=(1-\varphi)^{-1}$, $R(\varphi)=\varphi /(1-\varphi), \Phi(\rho)=\rho /(1+\rho), F_{\varphi}(k)=k, \xi(x)=\eta(x), M_{\varphi}(a)=(1-\varphi) /(1-$ $\left.\varphi e^{a}\right), R_{\varphi}(a)=\varphi e^{a} /\left[1-\varphi e^{a}\right]$ so that

$$
\begin{aligned}
I_{\varphi}(x) & =x \log \frac{x}{\varphi}-(1+x) \log (1+x)-\log (1-x) \\
& =x \log \frac{\Phi(x)}{\varphi}-\log \frac{Z(\Phi(x))}{Z(\varphi)} .
\end{aligned}
$$

We emphasize that formulas (3.2) and (3.3) have been deduced only in the case $g(k)=\mathbf{1}\{k \geq 1\}$, and may not hold in general.

For each $0<\varphi_{-}<\varphi_{+}$, define the entropy function $S_{\varphi_{-}, \varphi_{+}}: \mathbb{R} \rightarrow \mathbb{R}$ by

$$
S_{\varphi_{-}, \varphi_{+}}(E)=\lim _{\delta \rightarrow 0} \lim _{N \rightarrow \infty} \frac{1}{N} \log \sum_{\eta \in \Omega_{N}} \mathbf{1}\left\{\left|N^{-1} \log m_{\varphi_{-}, \varphi_{+}}^{N}(\eta)+E\right| \leq \delta\right\}
$$

whenever the limits exist. We may introduce in the sum $m_{\varphi_{-}, \varphi_{+}}^{N}(\eta)$ to get that the entropy function is equal to

$$
E+\lim _{\delta \rightarrow 0} \lim _{N \rightarrow \infty} \frac{1}{N} \log m_{\varphi_{-}, \varphi_{+}}^{N}\left\{\left|N^{-1} \log m_{\varphi_{-}, \varphi_{+}}^{N}(\eta)+E\right| \leq \delta\right\}
$$

Since $\log m_{\varphi_{-}, \varphi_{+}}^{N}(\eta)$ may be expressed in terms of the variables $\{\xi(x): 1 \leq x \leq$ $N-1\}$, which are independent under $m_{\varphi_{-}, \varphi_{+}}^{N}$, the large deviations principle gives that

$$
S_{\varphi_{-}, \varphi_{+}}(E)-E=-\inf _{\lambda} \int_{0}^{1} I_{\Phi(\bar{\rho}(x))}(\lambda(x)) d x
$$

where the infimum is carried over all profiles $\lambda:[0,1] \rightarrow \mathbb{R}$ such that

$$
\int_{0}^{1} \lambda(x) \log \Phi(\bar{\rho}(x))-\log Z(\Phi(\bar{\rho}(x))) d x=-E
$$

In view of (3.3), (3.2), in the case where $g(k)=\mathbf{1}\{k \geq 1\}$, the entropy function becomes

$$
S_{\varphi_{-}, \varphi_{+}}(E)-E=-\inf _{\rho} \int_{0}^{1} \rho(x) \log \frac{\Phi(\rho(x))}{\Phi(\bar{\rho}(x))}-\log \frac{Z(\Phi(\rho(x)))}{Z(\Phi(\bar{\rho}(x)))} d x,
$$

where the infimum is carried over all density profiles $\rho:[0,1] \rightarrow \mathbb{R}_{+}$such that $\mathcal{H}_{\varphi_{-}, \varphi_{+}}(\rho)=-E$. Therefore, in the case $g(k)=\mathbf{1}\{k \geq 1\}$, where an explicit formula is available, up to a linear term, $S_{\varphi_{-}, \varphi_{+}}(E)$ is obtained by minimizing the free energy functional $V_{\varphi_{-}, \varphi_{+}}$over all density profiles $\rho$ with energy $\mathcal{H}_{\varphi_{-}, \varphi_{+}}(\rho)$ equal to $-E$.

Finally, if we define $\widehat{S}_{\varphi_{-}, \varphi_{+}}: \mathbb{R} \rightarrow \mathbb{R}$ by

$$
\widehat{S}(E)=-\inf \left\{\int_{0}^{1} \rho \log \frac{\Phi(\rho)}{\Phi(\bar{\rho})}-\log \frac{Z(\Phi(\rho))}{Z(\Phi(\bar{\rho}))} d x: \int_{0}^{1}[\rho-\bar{\rho}] \log \Phi(\bar{\rho}) d x=-E\right\},
$$

we obtain that

$$
S_{\varphi_{-}, \varphi_{+}}(E)=E+\widehat{S}_{\varphi_{-}, \varphi_{+}}\left(E+\int_{0}^{1}\{\bar{\rho} \log \Phi(\bar{\rho})-\log Z(\Phi(\bar{\rho}))\} d x\right) .
$$


Note that $\widehat{S}(E) \leq 0$ and $\widehat{S}(0)=0$. As above, we stress that the identity (3.4) and all formulas thereafter were derived in the case $g(k)=\mathbf{1}\{k \geq 1\}$.

\section{The NONEQUilibrium PRESSURE}

We prove in this section Lemma 2.1. Recall the definition of the function $P$ introduced in (2.6). We first prove that $P$ is strictly concave. A long and tedious computation concluded with the change of variables $t=x^{\theta} /\left[x^{\theta}+(1-x)^{\theta}\right]$ shows that for $\theta \neq 0$,

$$
\begin{aligned}
P^{\prime \prime}(\theta) & =\frac{1}{\theta^{3}} \int_{A}^{B} s(t)^{2} \mu_{\theta}(d t)-\frac{1}{\theta^{3}}\left(\int_{A}^{B} s(t) \mu_{\theta}(d t)\right)^{2} \\
& -\frac{1}{\theta^{2}} \int_{A}^{B} t(1-t)\left(\log \frac{t}{1-t}\right)^{2} \mu_{\theta}(d t),
\end{aligned}
$$

where $A=\alpha^{\theta} /\left[\alpha^{\theta}+(1-\alpha)^{\theta}\right], B=\beta^{\theta} /\left[\beta^{\theta}+(1-\beta)^{\theta}\right]$,

$$
\mu_{\theta}(d t):=\frac{1}{Z(\theta)} m_{\theta}(t) d t, \quad m_{\theta}(t):=\frac{1}{t(1-t)} \frac{1}{\frac{1}{t^{1 / \theta}}+\frac{1}{(1-t)^{1 / \theta}}},
$$

and $Z(\theta)$ is a normalizing constant which makes $\mu_{\theta}$ a probability measure on $[A, B]$.

By Schwarz inequality, the first line of the expression of $P^{\prime \prime}$ without $\theta^{-3}$ is positive. Therefore, $P$ is strictly concave on the interval $(-\infty, 0)$. The strict concavity on the interval $(0, \infty)$ follows from the claim that for all $\theta>0,0<\alpha<\beta<1$,

$$
\int_{A}^{B} s(t)^{2} \mu_{\theta}(d t)-\left(\int_{A}^{B} s(t) \mu_{\theta}(d t)\right)^{2}<\theta \int_{A}^{B} t(1-t)\left(\log \frac{t}{1-t}\right)^{2} \mu_{\theta}(d t),
$$

It is enough to prove that

$$
\iint_{A \leq r \leq t \leq B}\{s(t)-s(r)\}^{2} \mu_{\theta}(d r) \mu_{\theta}(d t)<\theta \int_{A}^{B} t(1-t)\left(\log \frac{t}{1-t}\right)^{2} \mu_{\theta}(d t) .
$$

Let $H(u)=\theta^{-1}\left\{u^{-1 / \theta}+(1-u)^{-1 / \theta}\right\}$. Assume that $\theta \neq 1$ and denote by $R$ the primitive of $H$ given by $R(u)=(\theta-1)^{-1}\left\{u^{1-1 / \theta}-(1-u)^{1-1 / \theta}\right\}$. Hence, by Schwarz inequality, the left hand side of the previous inequality is bounded above by

$$
\begin{aligned}
& \iint_{A \leq r \leq t \leq B}\left(\int_{r}^{t}\left\{s^{\prime}(u)\right\}^{2} H(u)^{-1} d u\right)\left(\int_{r}^{t} H(v) d v\right) \mu_{\theta}(d r) \mu_{\theta}(d t) \\
& =\frac{1}{Z^{2}} \int_{A}^{B} d u\left\{s^{\prime}(u)\right\}^{2} H(u)^{-1} \int_{A}^{u} d r \int_{u}^{B} d t m_{\theta}(r) m_{\theta}(t)\{R(t)-R(r)\} .
\end{aligned}
$$

Since $s^{\prime}(r)=\log [r /(1-r)]$, to conclude the proof of Claim (4.2) for $\theta \neq 1$, it remains to show that

$$
\frac{1}{Z} \int_{A}^{u} d r \int_{u}^{B} d t m_{\theta}(r) m_{\theta}(t)\{R(t)-R(r)\}<1
$$

for all $\theta>0, \theta \neq 1$ and $0<A \leq u \leq B<1$.

The left hand side of the previous inequality can be written as

$$
\int_{u}^{B} m_{\theta}(t) R(t) d t \frac{1}{Z} \int_{A}^{u} m_{\theta}(r) d r-\int_{A}^{u} m_{\theta}(r) R(r) d r \frac{1}{Z} \int_{u}^{B} m_{\theta}(t) d t .
$$


We need to show that this expression is strictly bounded above by 1 for $A \leq u \leq B$. Let $K$ be a primitive of $m_{\theta} R$ and rewrite the previous expression as

$$
\begin{aligned}
J_{A, B}(u): & =[K(B)-K(u)] M(u)-[K(u)-K(A)][1-M(u)] \\
& =K(B) M(u)+K(A)[1-M(u)]-K(u),
\end{aligned}
$$

where $M(u)=Z^{-1} \int_{[A, u]} m_{\theta}(r) d r$. This expression represents the difference between the convex combination of $K(A)$ and $K(B)$, with weights $M(u), 1-M(u)$, and $K(u)$. For $A \leq u \leq B$, this difference is clearly absolutely bounded by the variation of $K$ on the interval $[A, B]$ :

$$
\sup _{A \leq u \leq B}\left|J_{A, B}(u)\right| \leq \sup _{A \leq v \leq B} K(v)-\inf _{A \leq v \leq B} K(v) .
$$

Maximizing over $0 \leq A \leq B \leq 1$, we get that

$$
\sup _{0 \leq A \leq u \leq B \leq 1}\left|J_{A, B}(u)\right| \leq \sup _{0 \leq v \leq 1} K(v)-\inf _{0 \leq v \leq 1} K(v) .
$$

A simple computation shows that

$$
K(t)=-\frac{\theta}{\theta-1} \log \left\{t^{1 / \theta}+(1-t)^{1 / \theta}\right\} .
$$

In particular, for $\theta \neq 1, K(0)=K(1)=0, K$ is symmetric around $1 / 2, K^{\prime}(1 / 2)=0$, $K$ decreases on the interval $[0,1 / 2]$ and increases on the interval $[1 / 2,1]$. Its total variation on the interval $[0,1]$ is therefore equal to $-K(1 / 2)=[\theta /(\theta-$ 1)] $\log 2^{1-1 / \theta}=\log 2<1$. This proves (4.3) and therefore Claim (4.2) for $\theta \neq 1$. The proof for $\theta=1$ is identical, the only difference being the explicit expression for the primitives.

The behavior of $P$ in a neighborhood of 0 is obtained through a simple Taylor expansion of the integrand. We have

$$
P(\theta)=-\log 2+\theta \log I_{1}-\theta^{2} \frac{I_{2}}{I_{1}}+O\left(\theta^{3}\right),
$$

where

$$
I_{1}=\frac{1}{\beta-\alpha} \int_{\alpha}^{\beta} \frac{d x}{\sqrt{x(1-x)}}
$$

and

$$
I_{2}=\frac{1}{2(\beta-\alpha)} \int_{\alpha}^{\beta} \frac{[\log x]^{2}+[\log (1-x)]^{2}}{\sqrt{x(1-x)}} d x .
$$

This completes the proof of the strict concavity of $P$ on $\mathbb{R}$.

We now turn to the claim that

$$
\lim _{\theta \rightarrow \pm \infty}\left\{P(\theta)-\theta E_{\mp}\right\}=0 .
$$

We consider the limit $\theta \uparrow \infty$, the other one being similar. By definition of $P$, we have to prove that

$$
\lim _{\theta \rightarrow \infty} \theta\left\{\log \left(\frac{A(\theta)}{\beta-\alpha}\right)-E_{-}\right\}=0 .
$$

A preliminary computation shows that $\lim _{\theta \rightarrow \infty} \log [A(\theta) /(\beta-\alpha)]=E_{-}$.

The proof of (4.4) depends on the positions of $\alpha$ and $\beta$ with respect to $1 / 2$, the most difficult case being when $0<\alpha \leq 1 / 2 \leq \beta<1$. Write $A(\theta)$ as

$$
\int_{\alpha}^{1 / 2} \frac{d x}{1-x} \exp \left\{-\frac{1}{\theta} \log \left[1+\left(\frac{x}{1-x}\right)^{\theta}\right]\right\}+\int_{1 / 2}^{\beta} \frac{d x}{x} \exp \left\{-\frac{1}{\theta} \log \left[1+\left(\frac{1-x}{x}\right)^{\theta}\right]\right\} .
$$


We concentrate on the first integral. Since $\left|e^{q}-1-q\right| \leq\left(q^{2} / 2\right) e^{|q|}, q \in \mathbb{R}$, the first integral is equal to

$$
\int_{\alpha}^{1 / 2} \frac{d x}{1-x}-\frac{1}{\theta} \int_{\alpha}^{1 / 2} \frac{d x}{1-x} \log \left[1+\left(\frac{x}{1-x}\right)^{\theta}\right]+\frac{1}{\theta^{2}} \varepsilon(\theta),
$$

where $\varepsilon(\theta)$ is a remainder absolutely bounded by $[\log 2]^{2}$ for $\theta>1$. The second integral in this expression vanishes as $\theta \uparrow \infty$ by the dominated convergence theorem. Hence, $\log [A(\theta) /(\beta-\alpha)]=E_{-}+o\left(\theta^{-1}\right)$, where $\theta o\left(\theta^{-1}\right)$ vanishes as $\theta \uparrow \infty$. This proves (4.4).

We finally consider the last statement of the lemma. By (2.7) and by the change of variables $x=u^{\theta}, P(\theta)-\theta P^{\prime}(\theta)$ is equal to

$$
\begin{aligned}
& \frac{1}{\theta A(\theta)} \int_{(\alpha /(1-\alpha))^{\theta}}^{(\beta /(1-\beta))^{\theta}} \frac{1}{1+x} \frac{x \log x-(1+x) \log (1+x)}{\left(x^{1 / \theta}+1\right)\left(x^{\theta-1}+x^{\theta}\right)^{1 / \theta}} d x \\
& \quad=\frac{1}{A(\theta)} \int_{\alpha / 1-\alpha}^{\beta / 1-\beta} \frac{d u}{(1+u)} \frac{u^{\theta} \log u^{\theta}-\left(1+u^{\theta}\right) \log \left(1+u^{\theta}\right)}{\left(1+u^{\theta}\right)^{1+[1 / \theta]}} .
\end{aligned}
$$

We examine the case $\theta \uparrow \infty, 0<\alpha \leq 1 / 2 \leq \beta$, the other ones being simpler. Since $A(\theta)$ converges to a constant as $\theta \uparrow \infty$, only the integral has to be estimated. By the dominated convergence theorem,

$$
\lim _{\theta \rightarrow \infty} \int_{\alpha / 1-\alpha}^{1} \frac{d u}{(1+u)} \frac{u^{\theta} \log u^{\theta}-\left(1+u^{\theta}\right) \log \left(1+u^{\theta}\right)}{\left(1+u^{\theta}\right)^{1+[1 / \theta]}}=0
$$

because the numerator vanishes as $\theta \uparrow \infty$. On the other hand, the integral in the interval $[1, \beta /(1-\beta)]$ can be written as

$$
-\int_{1}^{\beta / 1-\beta} \frac{d u}{u(1+u)} \frac{\log \left(1+u^{-\theta}\right)+u^{-\theta} \log \left(1+u^{\theta}\right)}{\left(1+u^{-\theta}\right)^{1+[1 / \theta]}} .
$$

By the dominated convergence theorem, this expression vanishes as $\theta \uparrow \infty$. This concludes the proof of the lemma.

\section{ENERGY BAND}

In this section, we determine the energy band $\left[E_{-}, E_{+}\right]$, i.e. the range of $V+\mathbb{S}$. Recall that $\mathcal{M}$ is the set of profiles $m:[0,1] \rightarrow[0,1]$ and let $\mathcal{M}_{+}, \mathcal{M}_{-}$be the set of profiles $m$ of the form $m(x)=\mathbf{1}\left\{\left[0, x_{0}\right]\right\}, m(x)=\mathbf{1}\left\{\left[x_{0}, 1\right]\right\}$, respectively, for some $x_{0} \in[0,1]$.

Lemma 5.1. For every $0<\alpha \leq \beta<1$,

$$
\sup _{m \in \mathcal{M}}\{V(m)+\mathbb{S}(m)\}=\sup _{m \in \mathcal{M}_{+}}\{V(m)+\mathbb{S}(m)\}=E_{+},
$$

and the supremum is attained for a unique profile $m \in \mathcal{M}_{+}$.

Proof. By [3, (2.11)],

$$
V(m)+\mathbb{S}(m)=\sup _{F \in \mathcal{F}} \mathcal{G}(m, F)
$$

where

$$
\mathcal{G}(m, F)=-\int_{0}^{1}\left\{m(x) \log F(x)+[1-m(x)] \log [1-F(x)]-\log \frac{F^{\prime}(x)}{\beta-\alpha}\right\} d x,
$$


and $\mathcal{F}$ is the set of all $C^{1}$ increasing functions $F:[0,1] \rightarrow[0,1]$ with boundary conditions $F(0)=\alpha, F(1)=\beta$. Moreover, the supremum is achieved at the unique solution $F$ of the boundary value problem (2.4).

We claim that for all $F$ in $\mathcal{F}$,

$$
\sup _{m \in \mathcal{M}} \mathcal{G}(m, F)=\sup _{m \in \mathcal{M}_{+}} \mathcal{G}(m, F) .
$$

The first identity in (5.1) follows from this assertion. To check (5.3), fix $F$ in $\mathcal{F}$ and note that the supremum is achieved by $m=\mathbf{1}\left\{\left[0, x_{F}\right]\right\} \in \mathcal{M}_{+}$, where $x_{F}=\sup \{x \in[0,1] ; F(x) \leq 1 / 2\}$, because $F$ is increasing. Of course $x_{F}=1$ if $\beta \leq 1 / 2$ and $x_{F}=0$ if $\alpha \geq 1 / 2$.

We claim that the solutions of the variational problem (5.1) belong to $\mathcal{M}_{+}$. Assume that there exists $m_{0} \in \mathcal{M}$ such that $V\left(m_{0}\right)+\mathbb{S}\left(m_{0}\right)=\sup _{m \in \mathcal{M}}\{V(m)+\mathbb{S}(m)\}$. Let $F_{0}$ be the unique solution of the boundary value problem (2.4) associated to $m_{0}$, let $x_{0}=\sup \left\{x \in[0,1] ; F_{0}(x) \leq 1 / 2\right\}$, let $m_{1}=1\left\{\left[0, x_{0}\right]\right\}$, and let $F_{1}$ be the solution of (2.4) associated to $m_{1}$. By (5.2),

$$
\mathcal{G}\left(m_{1}, F_{0}\right) \leq \mathcal{G}\left(m_{1}, F_{1}\right)=V\left(m_{1}\right)+\mathbb{S}\left(m_{1}\right)
$$

By definition of $m_{0}$ and by (5.2), the previous expression is bounded above by

$$
\sup _{m \in \mathcal{M}}\{V(m)+\mathbb{S}(m)\}=\mathcal{G}\left(m_{0}, F_{0}\right) \leq \sup _{m \in \mathcal{M}} \mathcal{G}\left(m, F_{0}\right)=\mathcal{G}\left(m_{1}, F_{0}\right),
$$

where the last identity follows from the argument presented in the previous paragraph. Since the first and the last terms in this sequence of inequalities are the same, all terms are equal and $\mathcal{G}\left(m_{1}, F_{0}\right)=\mathcal{G}\left(m_{1}, F_{1}\right)$. Since, by (5.2), $\sup _{F} \mathcal{G}\left(m_{1}, F\right)$ is uniquely attained at $F=F_{1}, F_{0}=F_{1}$. Therefore, in view of (2.4), $m_{1}=m_{0}$ a.s.

Let $m_{x_{0}}=\mathbf{1}\left\{\left[0, x_{0}\right]\right\}, x_{0} \in[0,1]$, and let $F$ be the solution of the nonlinear boundary value problem (2.4) with $m=m_{x_{0}}$. On the interval $\left[0, x_{0}\right], F(x)=\alpha e^{a x}$ for some $a>0$, and on the interval $\left[x_{0}, 1\right], F(x)=1-(1-\beta) e^{A(1-x)}$ for some $A>0$. Since $F$ must belong to $C^{1}([0,1]), a$ and $A$ satisfy

$$
\left\{\begin{array}{l}
\alpha a e^{a x_{0}}=(1-\beta) A e^{A\left(1-x_{0}\right)}, \\
\alpha e^{a x_{0}}=1-(1-\beta) e^{A\left(1-x_{0}\right)} .
\end{array}\right.
$$

Thus,

$$
e^{a x_{0}}=\frac{A}{a+A} \frac{1}{\alpha}, \quad e^{A\left(1-x_{0}\right)}=\frac{a}{a+A} \frac{1}{1-\beta}
$$

and therefore,

$$
\frac{1}{a} \log \left\{\frac{A}{a+A} \frac{1}{\alpha}\right\}+\frac{1}{A} \log \left\{\frac{a}{a+A} \frac{1}{1-\beta}\right\}=1 .
$$

Moreover, since $x_{0}$ belongs to $[0,1], a, A$ must satisfy $\max \left\{1-\beta, 1-\alpha e^{a}\right\} \leq$ $a /(a+A) \leq \min \left\{1-\alpha,(1-\beta) e^{A}\right\}$. Let $f, g:[0,1] \rightarrow \mathbb{R}$ be given by $f(x)=\alpha e^{a x}$, $g(x)=1-(1-\beta) e^{A(1-x)}$ and let $h(x)=f(x)-g(x)$. Since $h$ is convex and $0 \leq x_{0} \leq 1, h\left(x_{0}\right)=h^{\prime}\left(x_{0}\right)=0, h(0) \geq 0, h(1) \geq 0$. Hence, $1-\alpha \leq(1-\beta) e^{A}$, $1-\alpha e^{a} \leq 1-\beta$ so that

$$
1-\beta \leq \frac{a}{a+A} \leq 1-\alpha
$$


By the explicit expression of $F$ and by (5.4), (5.5), $\mathbb{S}\left(m_{x_{0}}\right)+V\left(m_{x_{0}}\right)$ is equal to

$$
\begin{aligned}
& \int_{0}^{1} \log \left\{\frac{F^{\prime}(x)}{\beta-\alpha}\right\} d x-\int_{0}^{x_{0}} \log F(x) d x-\int_{x_{0}}^{1} \log (1-F(x)) d x \\
& =\frac{1}{a} \log \left(\frac{A}{a+A} \frac{1}{\alpha}\right) \log a+\frac{1}{A} \log \left(\frac{a}{a+A} \frac{1}{1-\beta}\right) \log A-\log (\beta-\alpha) .
\end{aligned}
$$

Therefore, by the concavity of the log function and by (5.4),

$$
\begin{aligned}
\mathbb{S}\left(m_{x_{0}}\right)+V\left(m_{x_{0}}\right) & \leq \log \left\{\log \left(\frac{A}{A+a} \frac{1}{\alpha}\right)+\log \left(\frac{a}{a+A} \frac{1}{1-\beta}\right)\right\}-\log (\beta-\alpha) \\
& =\log \left\{\log \left[\frac{a}{a+A}\left(1-\frac{a}{a+A}\right) \frac{1}{\alpha(1-\beta)}\right]\right\}-\log (\beta-\alpha)
\end{aligned}
$$

By (5.6), the previous expression is bounded by

$$
\sup _{p \in[1-\beta, 1-\alpha]} \log \left\{\log \left[\frac{p(1-p)}{\alpha(1-\beta)}\right]\right\}-\log (\beta-\alpha)=E_{+},
$$

where the last identity follows by a direct computation. The last supremum is realized for $p=1-\beta$ if $\beta \leq 1 / 2$, for $p=1 / 2$ if $\alpha \leq 1 / 2 \leq \beta$ and for $p=1-\alpha$ if $1 / 2 \leq \alpha$

Up to this point, we proved that $\sup _{m \in \mathcal{M}}\{\mathbb{S}(m)+V(m)\} \leq E_{+}$. Assume that $\beta \leq 1 / 2$ and set $x_{0}=1$. In this case, by (5.4), $a /(a+A)=1-\beta$ and all inequalities in the previous argument are in fact identities. In particular, $\mathbb{S}\left(m_{1}\right)+V\left(m_{1}\right)=$ $\sup _{m \in \mathcal{M}}\{\mathbb{S}(m)+V(m)\}=E_{+}$. Moreover, since log is a strictly concave function and since by (5.4) $a /(a+A)>(1-\beta)$ for $x_{0}<1, \mathbb{S}\left(m_{1}\right)+V\left(m_{1}\right)>\mathbb{S}\left(m_{x_{0}}\right)+V\left(m_{x_{0}}\right)$ for $x_{0}<1$. In the same, way, if $\alpha \geq 1 / 2, \mathbb{S}\left(m_{0}\right)+V\left(m_{0}\right)=\sup _{m \in \mathcal{M}}\{\mathbb{S}(m)+V(m)\}=$ $E_{+}$and $\mathbb{S}\left(m_{0}\right)+V\left(m_{0}\right)>\mathbb{S}\left(m_{x_{0}}\right)+V\left(m_{x_{0}}\right)$ for $x_{0}>0$.

Finally, if $\alpha \leq 1 / 2 \leq \beta$, let $x=\log (2 \alpha) / \log [4 \alpha(1-\beta)]$ and observe that

$$
\mathbb{S}\left(m_{x}\right)+V\left(m_{x}\right)=\sup _{m \in \mathcal{M}}\{\mathbb{S}(m)+V(m)\}=E_{+}
$$

and that $\mathbb{S}\left(m_{x}\right)+V\left(m_{x}\right)>\mathbb{S}\left(m_{x_{0}}\right)+V\left(m_{x_{0}}\right)$ for $x_{0} \neq x$.

Lemma 5.2. For every $0<\alpha \leq \beta<1$,

$$
\inf _{m \in \mathcal{M}}\{V(m)+\mathbb{S}(m)\}=\inf _{m \in \mathcal{M}_{-}}\{V(m)+\mathbb{S}(m)\}=E_{-} .
$$

Proof. Recall (5.2). Since for each $F$ in $\mathcal{F}, \mathcal{G}(\cdot, F)$ is a continuous function for the weak topology, $V+\mathbb{S}$ is lower semicontinuous. In view of the explicit expression of $\mathcal{G}$ and by Jensen's inequality, $V+\mathbb{S}$ is bounded below. Hence, there exists $m_{0} \in \mathcal{M}$ such that $V\left(m_{0}\right)+\mathbb{S}\left(m_{0}\right)=\inf _{m \in \mathcal{M}}\{V(m)+\mathbb{S}(m)\}$.

Let $E_{-}^{*}=\inf _{m \in \mathcal{M}}\{V(m)+\mathbb{S}(m)\}=\inf _{m \in \mathcal{M}} \sup _{F \in \mathcal{F}} \mathcal{G}(m, F)$. This expression is bounded below by $\sup _{F \in \mathcal{F}} \inf _{m \in \mathcal{M}} \mathcal{G}(m, F)$. By the explicit expression of $\mathcal{G}$,

$$
\inf _{m \in \mathcal{M}} \mathcal{G}(m, F)=\mathcal{G}\left(m_{F}, F\right),
$$

where $m_{F}$ belongs to $\mathcal{M}_{-}$. If $\alpha \geq 1 / 2, \beta \leq 1 / 2, m_{F} \equiv 1, m_{F} \equiv 0$, respectively. Otherwise, $m_{F}=\mathbf{1}\left\{\left[x_{F}, 1\right]\right\}$, where $x_{F}$ is the unique point where $F$ is equal to $1 / 2$.

Assume that $\alpha \geq 1 / 2$. In this case, $E_{-}^{*} \geq \sup _{F \in \mathcal{F}} \mathcal{G}(\mathbf{1}, F)=V(\mathbf{1})+\mathbb{S}(\mathbf{1}) \geq E_{-}^{*}$. To conclude the proof of the lemma it remains to compute $\sup _{F \in \mathcal{F}} \mathcal{G}(\mathbf{1}, F)$ which can be done as in the previous lemma. The case $\beta \leq 1 / 2$ is similar. 
Assume that $\alpha<1 / 2<\beta$. In this case we have that $E_{-}^{*} \geq \sup _{F \in \mathcal{F}} \mathcal{G}\left(m_{F}, F\right)=$ $\sup _{0<x<1} \sup _{F \in \mathcal{F}_{x}} \mathcal{G}(\mathbf{1}\{[x, 1]\}, F)$, where $\mathcal{F}_{x}=\{F \in \mathcal{F}: F(x)=1 / 2\}$. Fix $0<x<$ 1. In each interval $[0, x],[x, 1]$, the variational problem $\sup _{F \in \mathcal{F}_{x}} \mathcal{G}(\mathbf{1}\{[x, 1]\}, F)$ is similar to the one in (5.2). In the interval $[0, x]$ the solution $F_{x, 0}$ of this variational problem solves the differential equation (2.4) with $m \equiv 0$ and boundary conditions $F(0)=\alpha, F(x)=1 / 2$. Analogously, in the interval $[x, 1]$ the solution $F_{x, 1}$ solves the differential equation (2.4) with $m \equiv 1$ and boundary conditions $F(x)=1 / 2$, $F(1)=\beta$. These solutions can be computed explicitly and one obtains that

$$
\begin{aligned}
& F_{x, 0}(y)=1-(1-\alpha) \exp \left\{-\frac{\log [2(1-\alpha)]}{x} y\right\}, \quad 0 \leq y \leq x, \\
& F_{x, 1}(y)=\beta \exp \left\{\frac{\log (2 \beta)}{1-x}(y-1)\right\}, \quad x \leq y \leq 1, \\
& \sup _{F \in \mathcal{F}_{x}} \mathcal{G}(\mathbf{1}\{[x, 1]\}, F)=x \log \frac{\log 2(1-\alpha)}{x}+(1-x) \log \frac{\log 2 \beta}{1-x}-\log (\beta-\alpha) .
\end{aligned}
$$

Maximizing over $x$ we deduce that $x^{*}=\log [2(1-\alpha)] / \log [4(1-\alpha) \beta]$ is the optimal value of $x$ and that

$$
E_{-}^{*} \geq \log \left\{\frac{1}{\beta-\alpha} \int_{\alpha}^{\beta} \frac{d x}{\max \{x, 1-x\}}\right\} .
$$

Moreover, a simple computation shows that for $F_{x^{*}, 0}^{\prime}\left(x^{*}\right)=F_{x^{*}, 1}^{\prime}\left(x^{*}\right)$. Hence, the function $G:[0,1] \rightarrow[\alpha, \beta]$ defined by $G(y)=F_{x^{*}, 0}(y) \mathbf{1}\left\{y \in\left[0, x^{*}\right]\right\}+$ $F_{x^{*}, 1}(y) \mathbf{1}\left\{y \in\left(x^{*}, 1\right]\right\}$ belongs to $\mathcal{F}$ and solves the boundary value problem (2.4) for $m=\mathbf{1}\left\{\left[x^{*}, 1\right]\right\}$. Hence, by definition of $E_{-}^{*}$ and by (5.2)

$$
\begin{aligned}
E_{-}^{*} \leq & \mathbb{S}\left(\mathbf{1}\left\{\left[x^{*}, 1\right]\right\}\right)+V\left(\mathbf{1}\left\{\left[x^{*}, 1\right]\right\}\right)=\mathcal{G}\left(\mathbf{1}\left\{\left[x^{*}, 1\right]\right\}, G\right) \\
& =\log \left\{\frac{1}{\beta-\alpha} \int_{\alpha}^{\beta} \frac{1}{\max \{x, 1-x\}}\right\} .
\end{aligned}
$$

This proves the lemma and shows that a profile with minimum energy is given by

$$
m_{\alpha, \beta}=\mathbf{1}\left\{\left[\frac{\log [2(1-\alpha)]}{\log [4(1-\alpha) \beta]}, 1\right]\right\} .
$$

We proved in the previous lemma that

$$
\inf _{m \in \mathcal{M}} \sup _{F \in \mathcal{F}} \mathcal{G}(m, F)=\sup _{F \in \mathcal{F}} \inf _{m \in \mathcal{M}} \mathcal{G}(m, F) .
$$

Fix $0<\alpha \leq \beta<1$ and assume that $V(m)+\mathbb{S}(m)=E_{-}^{*}$. Then,

$$
m(1-m)=0 \text { a. s. }
$$

Indeed, fix a profile $m$ in $\mathcal{M}$. Since $V(m)+\mathbb{S}(m)=\mathcal{G}(m, F)$, where $F$ is the solution of (2.4),

$$
\frac{\delta[V+\mathbb{S}](m)}{\delta m}=\frac{\delta \mathcal{G}(m, F)}{\delta m}+\frac{\delta \mathcal{G}(m, F)}{\delta F} \frac{\delta F}{\delta m} .
$$

Since $F$ solves the Euler equation (2.4) associated to the variational problem $\sup _{G} \mathcal{G}(m, G), \delta G(m, F) / \delta F=0$. On the other hand, $\delta \mathcal{G} / \delta m=-\log [F /(1-F)]$, so that

$$
\frac{\delta[V+\mathbb{S}](m)}{\delta m}=-\log \frac{F}{1-F} .
$$


This expression does not vanish because $F$ is strictly increasing. Therefore, the extremal values of $V+\mathbb{S}$ are attained at the boundary.

This formal argument can be made rigorous. By the proof of Theorem 7.1 in 6], $V$ and therefore $V+\mathbb{S}$ is Gâteaux differentiable, and the Gâteaux derivative of $V+\mathbb{S}$ at $m$ is equal to $-\log [F /(1-F)]$.

It follows from the previous results and the variational formula (2.5) that for $0<\alpha \leq \beta<1$,

$$
S(E)=-\infty \text { for } E \notin\left[E_{-}, E_{+}\right] \text {and } S\left(E_{ \pm}\right)=0 .
$$

\section{ISENTROPIC SURFACE}

We determine in this section the isentropic surfaces defined by

$$
\mathcal{E}_{K}=\left\{E \geq 0: S_{\alpha, \beta}(E)=K\right\}, \quad K \in[0, \log 2] .
$$

6.1. The equilibrium case. Assume that $\alpha=\beta$. We have already seen right after (2.3) that the energy band is reduced to the point $\log 2$ in the case $\alpha=1 / 2$. Assume therefore that $\alpha \neq 1 / 2$ and fix $K \in[0, \log 2)$. There exist exactly two solutions $0<m_{-}(K)<1 / 2<m_{+}(K)<1$ of $-s(m)=K$. Hence, in view of (2.3), the level set $\mathcal{E}_{K}=\left\{E_{-}(K), E_{+}(K)\right\}$, where

$$
E_{ \pm}(K)=-\log (1-\alpha)-\log \frac{\alpha}{1-\alpha} m_{ \pm}(K) .
$$

For $K=\log 2, \mathcal{E}_{K}$ is the singleton $\{-(1 / 2)[\log \alpha+\log (1-\alpha)]\}$.

6.2. The nonequilibrium case. Assume now that $\alpha<\beta$. Let $D: \mathbb{R} \rightarrow \mathbb{R}$ be given by $D(\theta)=\theta P^{\prime}(\theta)-P(\theta)$. Since $D^{\prime}(\theta)=\theta P^{\prime \prime}(\theta)$ and since, by Lemma 2.1 $P$ is strictly concave, $D$ is strictly increasing on $(-\infty ; 0]$ and strictly decreasing on $[0,+\infty)$. Moreover $D(0)=\log 2$ and, by Lemma 2.1, $\lim _{\theta \rightarrow \pm \infty} D(\theta)=0$. In particular, for every $K \in(0, \log 2)$ there exist exactly two values $\theta_{-}(K)<0<$ $\theta_{+}(K)$ such that $\theta_{ \pm}(K) P^{\prime}\left(\theta_{ \pm}(K)\right)-P\left(\theta_{ \pm}(K)\right)=K$.

Fix $0<K<\log 2$. By (2.13), $E$ belongs to $\mathcal{E}_{K}$ if and only if $K=\theta_{E} P^{\prime}\left(\theta_{E}\right)-$ $P\left(\theta_{E}\right)=D\left(\theta_{E}\right)$, where $P^{\prime}\left(\theta_{E}\right)=E$. Hence $\theta_{E}=\theta_{ \pm}(K)$ so that $E=P^{\prime}\left(\theta_{ \pm}(K)\right)$ and

$$
\mathcal{E}_{K}=\left\{P^{\prime}\left(\theta_{+}(K)\right), P^{\prime}\left(\theta_{-}(K)\right)\right\} .
$$

If we let $\alpha$ and $\beta$ vary, we see that the $K$-isentropic surface is composed of the two manifolds $E_{K}^{-}=P^{\prime}\left(\theta_{+}(K)\right)$ and $E_{K}^{+}=P^{\prime}\left(\theta_{-}(K)\right)$ which satisfy

$$
E_{K}^{-}(\alpha, \beta) \leq E_{K}^{+}(\alpha, \beta) \text {. }
$$

\section{COMPARISON With LOCAL EQUilibria}

In this section, we compare the entropy function $S_{\alpha, \beta}$ with the entropy function associated to product measures with a slowly varying density profile that will be called local equilibrium entropies.

Let $\nu_{\alpha, \beta}^{N}$ be the product probability measure on $\{0,1\}^{N-1}$ given by

$$
\nu_{\alpha, \beta}^{N}(\eta)=\prod_{x=1}^{N-1} \bar{\rho}(x / N)^{\eta_{x}}(1-\bar{\rho}(x / N))^{1-\eta_{x}}
$$


where $\bar{\rho}:[0,1] \rightarrow[\alpha, \beta]$ is the stationary profile $\bar{\rho}(x)=(1-x) \alpha+x \beta$. Denote by $\tilde{S}:=\tilde{S}_{\alpha, \beta}: \mathbb{R}_{+} \rightarrow\{-\infty\} \cup[0, \log 2]$ the entropy function corresponding to the Gibbs state $\nu_{\alpha, \beta}^{N}$ :

$$
\tilde{S}(E)=\lim _{\delta \rightarrow 0} \lim _{N \rightarrow \infty} \frac{1}{N} \log \sum_{\eta \in \Omega_{N}} \mathbf{1}\left\{\left|N^{-1} \log \nu_{\alpha, \beta}^{N}(\eta)+E\right| \leq \delta\right\}
$$

whenever the limits exist.

Let $\tilde{P}:=\tilde{P}_{\alpha, \beta}: \mathbb{R} \rightarrow \mathbb{R}$ be the function defined by

$$
\tilde{P}(\theta)=\frac{1}{\beta-\alpha} \int_{\alpha}^{\beta} \log \left(\frac{1}{x^{\theta}+(1-x)^{\theta}}\right) d x
$$

Lemma 7.1. $\tilde{P}$ is a $C^{2}$ strictly concave function and

$$
\lim _{\theta \rightarrow \pm \infty} \frac{\tilde{P}(\theta)}{\theta}=\tilde{E}_{\mp}, \quad \lim _{\theta \rightarrow \pm \infty}\left\{\tilde{P}(\theta)-\theta \tilde{P}^{\prime}(\theta)\right\}=0
$$

where

$$
\tilde{E}_{-}=\frac{1}{\beta-\alpha} \int_{\alpha}^{\beta} \log \frac{1}{\max \{x, 1-x\}} d x, \quad \tilde{E}_{+}=\frac{1}{\beta-\alpha} \int_{\alpha}^{\beta} \log \frac{1}{\min \{x, 1-x\}} d x .
$$

Moreover, as $\theta \rightarrow 0$,

$$
\tilde{P}(\theta)=-\log 2+\frac{\theta}{\beta-\alpha} \int_{\alpha}^{\beta} \log \frac{1}{\sqrt{x(1-x)}} d x+O\left(\theta^{2}\right)
$$

The proof is elementary and left to the reader. It follows from this result that

$$
\lim _{\theta \rightarrow \pm \infty} \tilde{P}^{\prime}(\theta)=\tilde{E}_{\mp}
$$

Proposition 7.2. For $E \geq 0$,

$$
\tilde{S}(E)=\inf _{\theta \in \mathbb{R}}\{\theta E-\tilde{P}(\theta)\} .
$$

If $E$ belongs to the energy band $\left(\tilde{E}_{-}, \tilde{E}_{+}\right)$, the infimum is attained at $\tilde{\theta}_{E}=\tilde{\theta}_{E}(\alpha, \beta)$ the unique solution of $\tilde{P}^{\prime}(\theta)=E$ and $\tilde{S}(E)=\tilde{\theta}_{E} E-\tilde{P}\left(\tilde{\theta}_{E}\right) . \quad \tilde{S}(E)=-\infty$ if $E \notin\left[\tilde{E}_{-}, \tilde{E}_{+}\right]$and $\tilde{S}\left(\tilde{E}_{ \pm}\right)=0$.

Proof. Multiplying and dividing the indicator in (7.1) by $2^{N-1}$, we reduce the computation of the entropy to a large deviations problem for independent Bernoulli random variables and we obtain that

$$
\tilde{S}(E)=\sup _{m \in \mathcal{M}}\{\mathbb{S}(m): I(m)+\mathbb{S}(m)=E\}
$$

where $I$ stands for the large deviations rate function given by

$$
I(m)=\int_{0}^{1}\left\{m(x) \log \frac{m(x)}{\bar{\rho}(x)}+[1-m(x)] \log \frac{1-m(x)}{1-\bar{\rho}(x)}\right\} d x .
$$

One should compare this expression with the variational formula (2.5) for the nonequilibrium entropy.

Repeating the arguments presented in the proof of (2.9), we deduce that

$$
\tilde{S}(E)=\mathbb{S}\left(\frac{\bar{\rho}^{\theta}}{(1-\bar{\rho})^{\theta}+\bar{\rho}^{\theta}}\right),
$$


where $\theta$ is the unique solution of $\tilde{P}^{\prime}(\theta)=E$. The rest of the proof is similar to the one of (2.13).

Let $E_{0}=P^{\prime}(0), \tilde{E}_{0}=\tilde{P}^{\prime}(0)$. By Lemma 2.1 and 7.1

$$
E_{0}=\log \left(\frac{1}{\beta-\alpha} \int_{\alpha}^{\beta} \frac{1}{\sqrt{x(1-x)}} d x\right), \quad \tilde{E}_{0}=\frac{1}{\beta-\alpha} \int_{\alpha}^{\beta} \log \left(\frac{1}{\sqrt{x(1-x)}}\right) d x .
$$

By Jensen's inequality, $\tilde{E}_{-}<E_{-}, \tilde{E}_{+}<E_{+}$and $\tilde{E}_{0}<E_{0}$. Since $\min \{x, 1-x\} \leq$ $1 / 2 \leq \max \{x, 1-x\}$ and $\sqrt{x(1-x)} \leq 1 / 2$, we may compare all variables with $\log 2$ to obtain in the end that $\tilde{E}_{-}<E_{-}<\log 2<\tilde{E}_{0}<\min \left\{E_{0}, \tilde{E}_{+}\right\} \leq \max \left\{E_{0}, \tilde{E}_{+}\right\}<$ $E_{+}$in the case $\alpha<\beta$.

The nonequilibrium and the local equilibrium entropy differ. For every $0<\alpha<$ $\beta<1, S<\tilde{S}$ in the interval $\left(E_{-}, \tilde{E}_{0}\right)$ and $\tilde{S}<S$ in the interval $\left(E_{0}, \tilde{E}_{+}\right)$.

Indeed, fix $E \in\left(E_{-}, \tilde{E}_{0}\right)$. By Jensen's inequality, $\theta^{-1} \tilde{P}(\theta)<\theta^{-1} P(\theta), \theta \in \mathbb{R}$. Therefore, for every $\theta>0, \theta E-P(\theta)<\theta E-\tilde{P}(\theta)$. On the other hand, since $E<E_{0}$ and $\theta_{E_{0}}=0, P^{\prime}\left(\theta_{E}\right)=E<E_{0}=P^{\prime}\left(\theta_{E_{0}}\right)=P^{\prime}(0)$. Hence, since $P^{\prime}$ decreases, $\theta_{E}>0$. A similar argument shows that $\tilde{\theta}_{E}>0$. In conclusion, by the variational formula for the entropies presented in (2.13) and 7.2

$$
\begin{aligned}
S(E) & =\inf _{\theta \in \mathbb{R}}\{\theta E-P(\theta)\}=\inf _{\theta>0}\{\theta E-P(\theta)\} \\
& <\inf _{\theta>0}\{\theta E-\tilde{P}(\theta)\}=\inf _{\theta \in \mathbb{R}}\{\theta E-\tilde{P}(\theta)\}=\tilde{S}(E) .
\end{aligned}
$$

A similar argument proves the other claim.

Consider the sequence of random variables $Y_{N}(\eta)=-N^{-1} \log \mu_{\alpha, \beta}^{N}(\eta), \tilde{Y}_{N}(\eta)=$ $-N^{-1} \log \nu_{\alpha, \beta}^{N}(\eta)$ defined on the probability space $\Omega_{N}$ equipped with the probability measure $\mu_{\alpha, \beta}^{N}, \nu_{\alpha, \beta}^{N}$, respectively. By (2.1), the sequence $\left(Y_{N}: N \geq 1\right)$ satisfies a large deviations principle with convex rate function $J(E)=E-S(E)$. By similar reasons, the sequence $\left(\tilde{Y}_{N}: N \geq 1\right)$ satisfies a large deviations principle with convex rate function $\tilde{J}(E)=E-\tilde{S}(E)$.

Bahadoran [1] proved that $E_{\mu_{\alpha, \beta}^{N}}\left[Y_{N}\right]$ and $E_{\nu_{\alpha, \beta}^{N}}\left[\tilde{Y}_{N}\right]$ have the same limit given by the Gibbs-Shannon entropy

$$
\bar{E}=-\int_{0}^{1} s(\bar{\rho}(x)) d x
$$

where $\bar{\rho}(x)=\alpha+(\beta-\alpha) x$. This result can be recovered from ours.

Lemma 7.3. The nonnegative rate functions $J$ and $\tilde{J}$ vanish at the same and unique point

$$
\bar{E}=P^{\prime}(1)=\tilde{P}^{\prime}(1) .
$$

In particular, $Y_{N}$ under $\mu_{\alpha, \beta}^{N}$, and $\tilde{Y}_{N}$ under $\nu_{\alpha, \beta}^{N}$ converge in probability to $\bar{E}$.

Proof. By Lemma 2.1 the variational formula (2.13) and the assertions following this formula, the nonnegative rate function $J$ is strictly convex on $\left[E_{-}, E_{+}\right]$, differentiable in $\left(E_{-}, E_{+}\right)$, and equal to $+\infty$ outside of the interval $\left[E_{-}, E_{+}\right]$. It has therefore a unique minimum $\bar{E} \in\left[E_{-}, E_{+}\right]$.

By (2.13) and (2.8), $S^{\prime}(E)=\theta_{E}$ on $\left(E_{-}, E_{+}\right)$, where $\theta_{E}$ is the unique solution of $P^{\prime}(\theta)=E$. By Lemma 2.1. $\lim _{\theta \rightarrow \pm \infty} P^{\prime}(\theta)=E_{\mp}$. It follows from the previous two facts that $\lim _{E \rightarrow E_{ \pm}} J^{\prime}(E)= \pm \infty$. Since $J$ is strictly convex, $J$ has a unique 
minimizer $\bar{E}$ in $\left(E_{-}, E_{+}\right)$solution of $\theta_{\bar{E}}=S^{\prime}(\bar{E})=1$. Applying $P^{\prime}$ on both sides of this equation, we deduce that $\bar{E}=P^{\prime}(1)$.

We claim that $J(\bar{E})=0$. To prove this identity we need to show that $S(\bar{E})=\bar{E}$ or, in view of (2.13), that $\theta_{\bar{E}} \bar{E}-P\left(\theta_{\bar{E}}\right)=\bar{E}$. Since $\theta_{\bar{E}}=1$, this equation is reduced to $P(1)=0$, which is easy to check in view of the explicit formula (2.6) for the nonequilibrium pressure.

The same argument applies to $\tilde{J}$ and the result follows from the identity

$$
P^{\prime}(1)=\tilde{P}^{\prime}(1)=-\int_{0}^{1} s(\alpha+(\beta-\alpha) x) d x .
$$

In [9, Section 7], the authors compute the limit of the variance of the sequences $\left(Y_{N}: N \geq 1\right)$ and $\left(\tilde{Y}_{N}: N \geq 1\right)$ and show that the limits differ. This result can be recovered from a second order expansion of the entropy function $S_{\alpha, \beta}$.

We have seen that the rate function $J$ has a unique minimum at $\bar{E}$. It is well known from the theory of large deviations that the asymptotic variance of the sequence $Y_{N}$ is given by $J^{\prime \prime}(\bar{E})^{-1}=-S^{\prime \prime}(\bar{E})^{-1}$. Since $\theta_{\bar{E}}=1$ and since $S$ is the Legendre transform of the nonequilibrium pressure $P$, we have that $S^{\prime \prime}(\bar{E})=$ $1 / P^{\prime \prime}\left(\theta_{\bar{E}}\right)=1 / P^{\prime \prime}(1)$. Hence, $-P^{\prime \prime}(1)$ is the asymptotic variance of the sequence $Y_{N}$.

By taking $\theta=1$ in (4.1) we obtain that

$$
\begin{aligned}
-P^{\prime \prime}(1) & =\frac{1}{\beta-\alpha} \int_{\alpha}^{\beta} t(1-t)\left[\log \left(\frac{t}{1-t}\right)\right]^{2} d t \\
& -\frac{1}{2(\beta-\alpha)^{2}} \int_{\alpha}^{\beta} d x \int_{\alpha}^{\beta} d y(s(x)-s(y))^{2} .
\end{aligned}
$$

A long and tedious computation shows that this expression coincides with the limiting variance derived in 9 .

A similar computation in the equilibrium model gives that the asymptotic variance of the sequence $\tilde{Y}_{N}$ is equal to

$$
\frac{1}{\beta-\alpha} \int_{\alpha}^{\beta} t(1-t)\left[\log \left(\frac{t}{1-t}\right)\right]^{2} d t
$$

In particular, the asymptotic variance in the nonequilibrium model is strictly bounded above by the asymptotic variance in the equilibrium model.

\section{Appendix A. The assumption $(\mathbf{H})$}

The stationary state $\mu_{\alpha, \beta}^{N}$ of the symmetric simple exclusion process with open boundary conditions can be expressed in terms of a product of matrices 9]: There exists matrices $D, E$ and vectors $|V\rangle,\langle W|$ such that

$$
\begin{gathered}
D E-E D=D+E, \quad\{(1-\beta) D-\beta E\}|V\rangle=|V\rangle, \\
\langle W|\{\alpha E-(1-\alpha) D\}=\langle W|
\end{gathered}
$$

and

$$
\mu_{\alpha, \beta}^{N}(\eta)=\frac{\omega_{N}(\eta)}{\left\langle W\left|(D+E)^{N-1}\right| V\right\rangle}
$$


where the weight $\omega_{N}(\eta)$ is given by

$$
\omega_{N}(\eta)=\left\langle W\left|\prod_{x=1}^{N-1}\{\eta(x) D+[1-\eta(x)] E\}\right| V\right\rangle .
$$

The partition function $\left\langle W\left|(D+E)^{N-1}\right| V\right\rangle$ can be computed explicitly [9, (3.11)]:

$$
\left\langle W\left|(D+E)^{N-1}\right| V\right\rangle=\frac{(N+1) !}{2(\beta-\alpha)^{N}} .
$$

Decompose the set $\{1, \ldots, N-1\}$ into $r=\varepsilon^{-1}$ adjacent intervals $K_{1}, \ldots, K_{r}$ of size $\varepsilon N$ and denote by $\mathbf{M}=\left(M_{1}, \ldots, M_{r}\right)$ the number of particles in each box. We recall that $\mu_{\alpha, \beta}^{N}(\cdot \mid \mathbf{M})$ is the probability measure $\mu_{\alpha, \beta}^{N}$ conditioned to have $M_{j}$ particles in $K_{j}, j=1, \ldots, r$.

Let $\eta^{+}, \eta^{-}$be the configuration in $\Omega_{N}(\mathbf{M})$ with all particles in each interval $K_{j}$ at the left most, right most positions, respectively. Hence, if $K_{j}=\left\{x_{1}, \ldots, x_{L}\right\}$, $M_{j}=M, \eta^{+}(x)=1$ if and only if $x_{1} \leq x \leq x_{M}, \eta^{-}(x)=1$ if and only if $x_{L-M+1} \leq x \leq x_{L}$.

Lemma A.1. For $0<\alpha \leq \beta<1, \eta \in \Omega_{N}(\mathbf{M})$,

$$
\mu\left(\eta^{-} \mid \mathbf{M}\right) \leq \mu(\eta \mid \mathbf{M}) \leq \mu\left(\eta^{+} \mid \mathbf{M}\right) .
$$

Proof. This is a simple consequence of the matrix product form of the stationary state. Let $\eta$ be any configuration in $\Omega_{N}$ and let $1 \leq x \leq N-1$ be any site such that $\eta(x)=1, \eta(x+1)=0$. Then, $\omega_{N}\left(\sigma^{x, x+1} \eta\right)-\omega_{N}(\eta)$ is equal to

$$
\begin{aligned}
& \left\langle W\left|\prod_{y=1}^{x-1}\{\eta(y) D+[1-\eta(y)] E\}[E D-D E] \prod_{y=x+2}^{N-1}\{\eta(y) D+[1-\eta(y)] E\}\right| V\right\rangle \\
& \quad=-\left\langle W\left|\prod_{y=1}^{x-1}\{\eta(y) D+[1-\eta(y)] E\}[D+E] \prod_{y=x+2}^{N-1}\{\eta(y) D+[1-\eta(y)] E\}\right| V\right\rangle \\
& \quad=-\omega_{N-1}(\xi)-\omega_{N-1}(\zeta) \leq 0,
\end{aligned}
$$

where $\xi, \zeta$ are the configuration of $\Omega_{N-1}$ given by $\xi=(\eta(1), \ldots, \eta(x-1), 1, \eta(x+$ $2), \ldots, \eta(N-1)), \zeta=(\eta(1), \ldots, \eta(x-1), 0, \eta(x+2), \ldots, \eta(N-1))$.

Hence, the derivation of the assumption $(\mathbf{H})$ is reduced to the proof that

$$
\lim _{\varepsilon \rightarrow 0} \limsup _{N \rightarrow \infty} \sup _{\mathbf{M}} \frac{1}{N}\left|\log \mu_{\alpha, \beta}^{N}\left(\eta^{+} \mid \mathbf{M}\right)-\log \mu_{\alpha, \beta}^{N}\left(\eta^{-} \mid \mathbf{M}\right)\right|=0 .
$$

or, equivalently, to the proof that

$$
\lim _{\varepsilon \rightarrow 0} \limsup _{N \rightarrow \infty} \sup _{\mathbf{M}} \frac{1}{N}\left|\log \mu_{\alpha, \beta}^{N}\left(\eta^{+}\right)-\log \mu_{\alpha, \beta}^{N}\left(\eta^{-}\right)\right|=0 .
$$

For each fixed $\varepsilon>0$ and $\mathbf{M}$, the configurations $\eta^{+}, \eta^{-}$are associated to density profiles $\rho^{+}, \rho^{-}:[0,1] \rightarrow[0,1]$ defined by $\rho^{+}=\sum_{1<i<r} 1\left\{\left[(i-1) \varepsilon,(i-1) \varepsilon+\rho_{i}\right)\right\}$, $\rho^{-}=\sum_{1 \leq i \leq r} 1\left\{\left[i \varepsilon-\rho_{i}, i \varepsilon\right)\right\}$, where $\rho_{i}=M_{i} / N, 1 \leq \bar{i} \leq r$ and $1\{A\}$ stands for the indicator of the set $A$. Therefore, by the large deviation principle for the density profiles under the stationary state $\mu_{\alpha, \beta}^{N}$ [9, 2, 7, 11,

$$
\lim _{N \rightarrow \infty} \frac{1}{N} \log \mu_{\alpha, \beta}^{N}\left(\eta^{ \pm}\right)=-V_{\alpha, \beta}\left(\rho^{ \pm}\right)
$$


where $V_{\alpha, \beta}$ is the functional introduced just before (2.4).

Hence, to prove assumption $(\mathbf{H})$, it remains to show that

$$
\lim _{\varepsilon \rightarrow 0} \sup _{\rho}\left|V_{\alpha, \beta}\left(\rho^{+}\right)-V_{\alpha, \beta}\left(\rho^{-}\right)\right|=0 .
$$

where the supremum is carried over all $0 \leq \rho_{i} \leq \varepsilon, 1 \leq i \leq r$. Since $\rho^{ \pm}(x)$ is either 0 or 1 , we may replace in the previous formula, $V_{\alpha, \beta}$ by $V_{\alpha, \beta}+\mathbb{S}$. By 3 , Theorem 4.1], this functional is continuous in $\mathcal{M}$. For each $\varepsilon>0$, denote by $\rho^{ \pm, \varepsilon}$ the profiles which attain the previous supremum with $V_{\alpha, \beta}$ by $V_{\alpha, \beta}+\mathbb{S}$. By compactness of $\mathcal{M}$, there exists a subsequence $\varepsilon_{k} \downarrow 0$ for which

$$
\begin{aligned}
& \lim _{\varepsilon \rightarrow 0} \sup _{\rho}\left|V_{\alpha, \beta}\left(\rho^{+}\right)-V_{\alpha, \beta}\left(\rho^{-}\right)\right| \\
& \quad=\lim _{k \rightarrow \infty}\left|V_{\alpha, \beta}\left(\rho^{+, \varepsilon_{k}}\right)+\mathbb{S}\left(\rho^{+, \varepsilon_{k}}\right)-V_{\alpha, \beta}\left(\rho^{-, \varepsilon_{k}}\right)-\mathbb{S}\left(\rho^{-, \varepsilon_{k}}\right)\right|
\end{aligned}
$$

and $\rho^{+, \varepsilon_{k}}$ converges weakly to some profile $\rho$. Clearly, the sequence $\rho^{-, \varepsilon_{k}}$ converges weakly to the same profile $\rho$. Since $V_{\alpha, \beta}+\mathbb{S}$ is continuous in $\mathcal{M}$, assumption $(\mathbf{H})$ is proved.

Acknowledgments. The authors thank B. Derrida for indicating to them Lemma A.1 L. Bertini, D. Gabrielli, G. Jona-Lasinio and J. L. Lebowitz for fruitful discussions and the referees for their remarks. The end of Section 7 , in particular, was added by suggestion of one of the referees. The warm hospitality of IMPA is also acknowledged by the first author.

\section{REFERENCES}

[1] Bahadoran, C., On the convergence of entropy for stationary exclusion processes with open boundaries, J. Stat. Phys. 126 (2007), no. 4-5, 1069-1082.

[2] Bertini L., De Sole A., Gabrielli D., Jona-Lasinio G., Landim C., Macroscopic fluctuation theory for stationary non equilibrium state. J. Statist. Phys. 107, 635-675 (2002).

[3] L. Bertini, A. De Sole, D. Gabrielli, G. Jona-Lasinio, C. Landim, Large deviations for the boundary driven symmetric simple exclusion process, Math. Phys. Anal. Geom. 6 (2003), no. 3, 231-267.

[4] Bertini L., De Sole A., Gabrielli D., Jona-Lasinio G., Landim C., Stochastic interacting particle systems out of equilibrium. J. Stat. Mech. P07014. (2007)

[5] Bertini, L.; De Sole, A.; Gabrielli, D.; Jona-Lasinio, G.; Landim, C. Towards a nonequilibrium thermodynamics: a self-contained macroscopic description of driven diffusive systems. J. Stat. Phys. 135 (2009), no. 5-6, 857-872.

[6] Bertini, L.; De Sole, A.; Gabrielli, D.; Jona-Lasinio, G.; Landim, C. Action functional and quasi-potential for the Burgers equation in a bounded interval. arXiv:1004.2225 (2010).

[7] Bodineau T., Giacomin G., From dynamic to static large deviations in boundary driven exclusion particles systems. Stoch. Proc. Appl. 110, 67-81 (2004).

[8] Derrida, B., Non-equilibrium steady states: fluctuations and large deviations of the density and of the current, J. Stat. Mech. P07023 (2007)

[9] Derrida,B., Lebowitz, J.L., Speer, E.R., Large Deviation of the Density Profile in the Steady State of the Open Symmetric Simple Exclusion, J. Statist. Phys. 107 (2002), no. 3-4, 599-634.

[10] Derrida, B.; Lebowitz, J. L.; Speer, E. R.; Entropy of open lattice systems, J. Stat. Phys. 126 (2007), no. 4-5, 1083-1108.

[11] Farfan J.; Static large deviations of boundary driven exclusion processes. Preprint 2009, arXiv:0908.1798v1

[12] Presutti, E., Scaling limits in statistical mechanics and microstructures in continuum mechanics. Theoretical and Mathematical Physics. Springer, Berlin, 2009. xvi+467 pp. ISBN: 978-3-540-73304-1

[13] H. Spohn, Long range correlations for stochastic lattice gases in a nonequilibrium steady state. J. Phys. A 16 (1983), 4275-4291. 
Université de Lyon and CNRS, UMPA, UMR-CNRS 5669, ENS-Lyon, 46, AlléE d’Italie, 69364 Lyon Cedex 07 - France.

E-MAIL: Cedric.Bernardin@umpa.ens-lyon.fr

impa, Estrada Dona Castorina 110, CeP 22460 Rio de Janeiro, Brasil and CNRS UMR 6085, Université de Rouen, Avenue de L'Université, BP.12, Technopôle du MadrilLet, F76801 Saint-Étienne-Du-Rouvray, France.

E-MAIL: landim@impa.br 